\title{
The Topp-Leone Odd Burr III-G Family of Distributions: Model, Properties and Applications
}

\author{
Thatayaone Moakofi , Broderick Oluyede , Morongwa Gabanakgosi * \\ Department of Mathematics and Statistical Sciences, Botswana International University of Science and Technology, Botswana
}

\begin{abstract}
We propose and investigate a new generalized family of distributions called the Topp-Leone Odd Burr III-G (TLOBIII-G) family of distributions. We present the sub-families of this new family of distributions. Properties of the new family of distributions includs sub-models, quantile function, moments, incomplete and probability weighted moments, distribution of the order statistics, and Rényi entropy are derived. The Maximum likelihood estimation technique is used to estimate the model parameters, and a Monte Carlo simulation study is employed to examine the performance of the model. Two real data sets are used to prove the importance of the TL-OBIII-G family of distributions.
\end{abstract}

Keywords Odd Burr III Distribution, Topp-Leone-G Distribution, Generalized Distribution, Maximum Likelihood Estimation

Mathematics Subject Classifications 60E05; 62F10; 62E15

DOI: $10.19139 /$ soic-2310-5070-1135

\section{Introduction}

The limitations of the well-known standard distribution in data modeling have motivated researchers to extend distributions by adding one or more shape parameters to gain flexibility in the new distribution. Some of the generated families in the literature including the odd Burr III family of distributions by Jamal et al. [19], the Nadarajah Haghighi Topp-Leone-G family of distributions by Reyad et al. [33], the Marshall-Olkin Odd Burr III-G family of distributions by Afify et al. [2], the generalized transmuted-G family proposed by Nofal et al. [28], Marshall-Olkin alpha power-G by Nassar et al. [27], beta-G by Eugene et al. [17], Topp Leone odd Lindley-G by Reyad et al. [32], odd Lomax-G by Cordeiro et al. [9], exponentiated Weibull-H by Cordeiro et al. [10], the Burr III Marshal Olkin family of distributions by Bhatti et al. [7]; among others.

The Burr III (BIII) distribution was one of the twelve cumulative distribution functions introduced by Burr [8]. In the actuarial literature it is referred to as the inverse Burr distribution (Klugman et al. [21]) and as the kappa distribution in the meteorological area [25]. The BIII distribution has been studied and also used in various fields of sciences. It has been used in finance, environmental studies, survival analysis, and reliability theory, see Lindsay et al. [24] and Gove et al. [18]. A random variable $X$ has the odd Burr III-G distribution if its cumulative distribution function (cdf) and probability density function (pdf) are given by

$$
F(x ; \alpha, \beta, \xi)=\left(1+\left(\frac{G(x ; \xi)}{\bar{G}(x ; \xi)}\right)^{-\alpha}\right)^{-\beta}
$$

\footnotetext{
*Correspondence to: Morongwa Gabanakgosi (Email:morongwa.gabanakgosi@ studentmail.biust.ac.bw). Department of Mathematics and Statistical Sciences, Botswana International University of Science and Technology, Palapye, Botswana.
}

ISSN 2310-5070 (online) ISSN 2311-004X (print)

Copyright (c) 2022 International Academic Press 
and

$$
f(x ; \alpha, \beta, \xi)=\alpha \beta\left(1+\left(\frac{G(x ; \xi)}{\bar{G}(x ; \xi)}\right)^{-\alpha}\right)^{-\beta-1}\left(\frac{G(x ; \xi)}{\bar{G}(x ; \xi)}\right)^{-\alpha-1} \frac{g(x ; \xi)}{(\bar{G}(x ; \xi))^{2}},
$$

for $\alpha, \beta>0$, and parameter vector $\xi$, respectively.

The Topp-Leone transformation was applied to several distributions, for example; Topp-Leone Inverse Weibull distribution by Abbas et al. [1], Type II Topp-Leone Inverse Rayleigh distribution introduced by Mohammed and Yahia [26], Topp-Leone Weibull-Lomax distribution presented by Jamal et al. [20], the Topp-Leone generalized exponential power series distribution by Kunjiratanachot et al. [22] and Aryal et al. [5] developed the Topp-Leone Generated Weibull distribution. Al-Shomrani et al. [3] proposed the Topp-Leone family of distributions with the cdf and pdf given by

$$
F_{T L-G}(x ; b, \xi)=\left[1-\bar{G}^{2}(x ; \xi)\right]^{b}
$$

and

$$
f_{T L-G}(x ; b, \xi)=2 \alpha b\left[1-\bar{G}^{2}(x ; \xi)\right]^{b-1} \bar{G}(x ; \xi) g(x ; \xi),
$$

respectively, for $b>0$ and parameter vector $\xi$.

The basic motivations for developing the TL-OBIII-G family of distributions are:

- to construct and generate distributions with symmetric, left-skewed, right-skewed, reversed-J shapes;

- to provide better fits than other generated distributions under the same transformation;

- to construct heavy-tailed distributions for modeling different real data sets.

The rest of the results are outlined as follows: Section 2 contain the new TL-OBIII-G family of distributions and its properties such as sub-families, hazard rate function (hrf), quantile function to mention a few. In Section 3 estimation of the parameters of the TL-OBIII-G family of distributions via the method of maximum likelihood is presented, followed by a Monte Carlo simulation study to examine the performance of the maximum likelihood estimates in section 4. Two applications to real data sets are given in section 5, followed by some concluding remarks in section 6

\section{The Model and Statistical Properties}

In this section, we derive the Topp-Leone Odd Burr III-G (TL-OBIII-G) family of distributions and its statistical properties.

\subsection{Topp-Leone Odd Burr III-G Family of Distributions}

Inserting the cdf of the OBIII-G distribution into equation (1), we obtain the new family of distribution herein referred to as Topp-Leone Odd Burr III-G family of distributions. The cdf and pdf of the proposed Topp-Leone Odd Burr III-G (TL-OBIII-G) family of distributions are given by

$$
F(x ; \alpha, \beta, b, \xi)=\left[1-\left(1-\left(1+\left(\frac{G(x ; \xi)}{\bar{G}(x ; \xi)}\right)^{-\alpha}\right)^{-\beta}\right)^{2}\right]^{b}
$$


and

$$
\begin{aligned}
f(x ; \alpha, \beta, b, \xi) & =2 \alpha \beta b\left[1-\left(1-\left(1+\left(\frac{G(x ; \xi)}{\bar{G}(x ; \xi)}\right)^{-\alpha}\right)^{-\beta}\right)^{2}\right]^{b-1} \\
& \times\left(1-\left(1+\left(\frac{G(x ; \xi)}{\bar{G}(x ; \xi)}\right)^{-\alpha}\right)^{-\beta}\right)\left(1+\left(\frac{G(x ; \xi)}{\bar{G}(x ; \xi)}\right)^{-\alpha}\right)^{-\beta-1} \\
& \times\left(\frac{G(x ; \xi)}{\bar{G}(x ; \xi)}\right)^{-\alpha-1} \frac{g(x ; \xi)}{(\bar{G}(x ; \xi))^{2}}
\end{aligned}
$$

respectively, for $\alpha, \beta, b>0$ and parameter vector $\xi$.

The hazard rate function (hrf) and quantile functions of the TL-OBIII-G family of distributions are respectively given below. The hrf is given by

$$
\begin{aligned}
h_{F}(x ; \alpha, \beta, b, \xi) & =2 \alpha \beta b\left[1-\left(1-\left(1+\left(\frac{G(x ; \xi)}{\bar{G}(x ; \xi)}\right)^{-\alpha}\right)^{-\beta}\right)^{2}\right]^{b-1} \\
& \times\left(1-\left(1+\left(\frac{G(x ; \xi)}{\bar{G}(x ; \xi)}\right)^{-\alpha}\right)^{-\beta}\right)\left(1+\left(\frac{G(x ; \xi)}{\bar{G}(x ; \xi)}\right)^{-\alpha}\right)^{-\beta-1} \\
& \times\left(\frac{G(x ; \xi)}{\bar{G}(x ; \xi)}\right)^{-\alpha-1} \frac{g(x ; \xi)}{(\bar{G}(x ; \xi))^{2}} \\
& \times\left(1-\left[1-\left(1-\left(1+\left(\frac{G(x ; \xi)}{\bar{G}(x ; \xi)}\right)^{-\alpha}\right)^{-\beta}\right)^{2}\right]^{-b}\right)^{-1}
\end{aligned}
$$

for $\alpha, \beta, \delta>0$ and parameter vector $\xi$.

The quantile function of the TL-OBIII-G family of distributions is obtained by solving the non-linear equation:

$$
F(x ; \alpha, \beta, b, \xi)=\left[1-\left(1-\left(1+\left(\frac{G(x ; \xi)}{\bar{G}(x ; \xi)}\right)^{-\alpha}\right)^{-\beta}\right)^{2}\right]^{b}=u,
$$

for $0 \leq u \leq 1$, that is,

$$
\frac{G(x ; \xi)}{\bar{G}(x ; \xi)}=\left(\left(1-\left(1-u^{\frac{1}{b}}\right)^{\frac{1}{2}}\right)^{\frac{-1}{\beta}}-1\right)^{\frac{-1}{\alpha}},
$$

so that

$$
G(x ; \xi)=\left(\left(\left(1-\left(1-u^{\frac{1}{b}}\right)^{\frac{1}{2}}\right)^{\frac{-1}{\beta}}-1\right)^{\frac{-1}{\alpha}}+1\right)^{-1} .
$$

Consequently, the quantile function for the TL-OBIII-G family of distributions is given by

$$
Q_{G}(u ; \alpha, \beta, b, \xi)=X=G^{-1}\left[\left(\left(\left(1-\left(1-u^{\frac{1}{b}}\right)^{\frac{1}{2}}\right)^{\frac{-1}{\beta}}-1\right)^{\frac{-1}{\alpha}}+1\right)^{-1}\right] \text {. }
$$


It follows therefore that random numbers can be generated from the TL-OBIII-G family of distributions based on equation (8).

\subsection{Sub-Families of TL-OBIII-G Family of Distributions}

In this subsection, some sub-families of the TL-OBIII-G family of distributions are presented.

- When $\alpha=1$, the Topp-Leone Odd Burr III-G (TL-OBIII-G) family of distributions reduces to a new family of distributions with the cdf

$$
F(x ; \beta, b, \xi)=\left[1-\left(1-\left(1+\left(\frac{G(x ; \xi)}{\bar{G}(x ; \xi)}\right)^{-1}\right)^{-\beta}\right)^{2}\right]^{b},
$$

for $\beta, b>0$, and parameter vector $\xi$.

- If $\beta=1$, we obtain the new family of distributions with the cdf

$$
F(x ; \alpha, b, \xi)=\left[1-\left(1-\left(1+\left(\frac{G(x ; \xi)}{\bar{G}(x ; \xi)}\right)^{-\alpha}\right)^{-1}\right)^{2}\right]^{b}
$$

for $\alpha, b>0$, and parameter vector $\xi$.

- If $b=1$ we obtain the new family of distributions with the cdf

$$
F(x ; \alpha, \beta, \xi)=\left[1-\left(1-\left(1+\left(\frac{G(x ; \xi)}{\bar{G}(x ; \xi)}\right)^{-\alpha}\right)^{-\beta}\right)^{2}\right]
$$

for $\alpha, \beta>0$ and parameter vector $\xi$.

- If $\beta=\alpha=1$ we obtain the new family of distributions with the cdf

$$
F(x ; b, \xi)=\left[1-\left(1-\left(1+\left(\frac{G(x ; \xi)}{\bar{G}(x ; \xi)}\right)^{-1}\right)^{-1}\right)^{2}\right]^{b},
$$

for $b>0$ and parameter vector $\xi$.

- If $\beta=b=1$ we obtain the new family of distributions with the cdf

$$
F(x ; \alpha, \xi)=\left[1-\left(1-\left(1+\left(\frac{G(x ; \xi)}{\bar{G}(x ; \xi)}\right)^{-\alpha}\right)^{-1}\right)^{2}\right],
$$

for $\alpha>0$ and parameter vector $\xi$.

- If $\beta=\alpha=b=1$ we obtain the new family of distributions with the cdf

$$
F(x ; \xi)=\left[1-\left(1-\left(1+\left(\frac{G(x ; \xi)}{\bar{G}(x ; \xi)}\right)^{-1}\right)^{-1}\right)^{2}\right],
$$

for parameter vector $\xi$.

\subsection{Some Specialized Sub Models}

In this subsection, we present sub-models of TL-OBIII-G family of distributions by specifying $G(x ; \xi)$ and $g(x ; \xi)$ in equations (3) and (4). 
2.3.1. TL-OBIII-LLoG Distribution If the baseline cdf and pdf are given by $G(x ; \lambda)=1-\left(1+x^{\lambda}\right)^{-1}$ and $g(x ; \lambda)=\lambda x^{\lambda-1}\left(1+x^{\lambda}\right)^{-2}$, for $\lambda>0$, and $x>0$, the cdf and pdf of Topp-Leone Odd Burr III-log-logistic (TLOBIII-LLoG) distribution are given by

$$
F(x ; \alpha, \beta, b, \lambda)=\left[1-\left(1-\left(1+\left(\frac{1-\left(1+x^{\lambda}\right)^{-1}}{\left(1+x^{\lambda}\right)^{-1}}\right)^{-\alpha}\right)^{-\beta}\right)^{2}\right]^{b}
$$

and

$$
\begin{aligned}
f(x ; \alpha, \beta, b, \lambda) & =2 \alpha \beta b\left[1-\left(1-\left(1+\left(\frac{1-\left(1+x^{\lambda}\right)^{-1}}{\left(1+x^{\lambda}\right)^{-1}}\right)^{-\alpha}\right)^{-\beta}\right)^{2}\right]^{b-1} \\
& \times\left(1-\left(1+\left(\frac{1-\left(1+x^{\lambda}\right)^{-1}}{\left(1+x^{\lambda}\right)^{-1}}\right)^{-\alpha}\right) \frac{g(x ; \xi)}{\left(\left(1+x^{\lambda}\right)^{-1}\right)^{2}}\right. \\
& \times\left(1+\left(\frac{1-\left(1+x^{\lambda}\right)^{-1}}{\left(1+x^{\lambda}\right)^{-1}}\right)^{-\alpha}\right)^{-\beta-1}\left(\frac{1-\left(1+x^{\lambda}\right)^{-1}}{\left(1+x^{\lambda}\right)^{-1}}\right)^{-\alpha-1},
\end{aligned}
$$

respectively, for $\alpha, \beta, b, \lambda>0$. The hrf is given by

$$
\begin{aligned}
h_{F}(x ; \alpha, \beta, b, \lambda) & =2 \alpha \beta b\left[1-\left(1-\left(1+\left(\frac{1-\left(1+x^{\lambda}\right)^{-1}}{\left(1+x^{\lambda}\right)^{-1}}\right)^{-\alpha}\right)^{-\beta}\right)^{2}\right]^{b-1} \\
& \times\left(1-\left(1+\left(\frac{1-\left(1+x^{\lambda}\right)^{-1}}{\left(1+x^{\lambda}\right)^{-1}}\right)^{-\alpha}\right) \frac{g(x ; \xi)}{\left(\left(1+x^{\lambda}\right)^{-1}\right)^{2}}\right. \\
& \times\left(1+\left(\frac{1-\left(1+x^{\lambda}\right)^{-1}}{\left(1+x^{\lambda}\right)^{-1}}\right)^{-\alpha}\right)^{-\beta-1}\left(\frac{1-\left(1+x^{\lambda}\right)^{-1}}{\left(1+x^{\lambda}\right)^{-1}}\right)^{-\alpha-1} \\
& \times\left(1-\left[1-\left(1-\left(1+\left(\frac{1-\left(1+x^{\lambda}\right)^{-1}}{\left(1+x^{\lambda}\right)^{-1}}\right)^{-\alpha}\right)^{-\beta}\right)^{2}\right]^{-1}\right.
\end{aligned}
$$



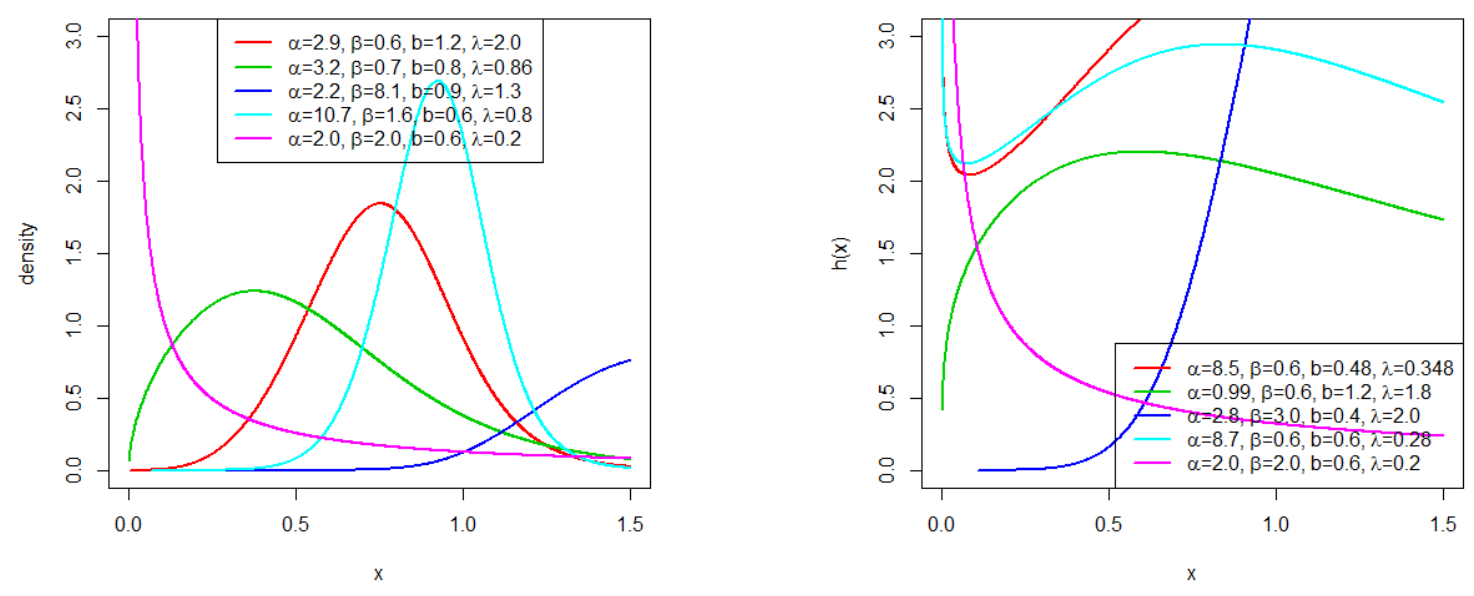

Figure 1. Density and Hazard Rate Function Plots for TL-OBIII-LLoG Distribution

Figure 1 shows the plots of pdf and hrf of the TL-OBIII-LLoG distribution, respectively. The pdf can take several shapes including increasing, right-skewed, left-skewed, unimodal and reverse-J shapes. The TL-OBIIILLoG hrf displays increasing, decreasing, bathtub, bathtub followed by upside-down bathtub, and upside-down bathtub shapes. These non-monotonic shapes are likely to be encountered in real life situations.

Table 1. Table of Quantile for TL-OBIII-LLoG Distribution

\begin{tabular}{c|ccccc}
\hline \multicolumn{5}{c}{$(\alpha, \beta, b, \lambda)$} \\
\hline$u$ & $(1.4,1.0,0.4,1.8)$ & $(0.6,1.3,2.2,1.0)$ & $(0.8,1.0,2.6,1.7)$ & $(2.0,1.0,1.0,2.0)$ & $(1.0,1.8,0.6,2.5)$ \\
\hline 0.1 & 0.0773 & 0.2157 & 0.4168 & 0.4799 & 0.3786 \\
0.2 & 0.1555 & 0.4281 & 0.5756 & 0.5885 & 0.5050 \\
0.3 & 0.2330 & 0.7007 & 0.7224 & 0.6643 & 0.6133 \\
0.4 & 0.3154 & 1.0671 & 0.8773 & 0.7354 & 0.7139 \\
0.5 & 0.4046 & 1.5836 & 1.0497 & 0.8022 & 0.8174 \\
0.6 & 0.5027 & 2.3724 & 1.2586 & 0.8735 & 0.9319 \\
0.7 & 0.6194 & 3.6896 & 1.5376 & 0.9544 & 1.0676 \\
0.8 & 0.7768 & 6.3088 & 1.9540 & 1.0557 & 1.2502 \\
0.9 & 1.0327 & 13.9007 & 2.7804 & 1.2141 & 1.5600 \\
\hline
\end{tabular}

2.3.2. TL-OBIII-Power Distribution The cdf and pdf of power distribution are given by $G(x ; \theta, k)=(\theta x)^{k}$ and $g(x ; \theta, k)=k \theta^{k} x^{k-1}$, for $\theta, k>0$, and $x \in\left(0, \frac{1}{\theta}\right)$. The Topp-Leone Odd Burr III-Power (TL-OBIII-P) distribution has cdf and pdf given by

$$
F(x ; \alpha, \beta, b, \theta, k)=\left[1-\left(1-\left(1+\left(\frac{(\theta x)^{k}}{1-(\theta x)^{k}}\right)^{-\alpha}\right)^{-\beta}\right)^{2}\right]^{b}
$$


and

$$
\begin{aligned}
f(x ; \alpha, \beta, b, \theta, k) & =2 \alpha \beta b\left[1-\left(1-\left(1+\left(\frac{(\theta x)^{k}}{1-(\theta x)^{k}}\right)^{-\alpha}\right)^{-\beta}\right]^{2}\right]^{b-1} \\
& \times\left(1-\left(1+\left(\frac{(\theta x)^{k}}{1-(\theta x)^{k}}\right)^{-\alpha}\right)^{-\beta}\right)\left(1+\left(\frac{(\theta x)^{k}}{1-(\theta x)^{k}}\right)^{-\alpha}\right)^{-\beta-1} \\
& \times\left(\frac{(\theta x)^{k}}{1-(\theta x)^{k}}\right)^{-\alpha-1} \frac{k \theta^{k} x^{k-1}}{\left(1-(\theta x)^{k}\right)^{2}}
\end{aligned}
$$

respectively, for $\alpha, \beta, b, \theta, k>0$. The hrf is given by

$$
\begin{aligned}
h_{F}(x ; \alpha, \beta, b, \theta, k) & =2 \alpha \beta b\left[1-\left(1-\left(1+\left(\frac{(\theta x)^{k}}{1-(\theta x)^{k}}\right)^{-\alpha}\right)^{-\beta}\right]^{2}\right]^{b-1} \\
& \times\left(1-\left(1+\left(\frac{(\theta x)^{k}}{1-(\theta x)^{k}}\right)^{-\alpha}\right)^{-\beta}\right)\left(1+\left(\frac{(\theta x)^{k}}{1-(\theta x)^{k}}\right)^{-\alpha}\right)^{-\beta-1} \\
& \times\left(\frac{(\theta x)^{k}}{1-(\theta x)^{k}}\right)^{-\alpha-1} \frac{k \theta^{k} x^{k-1}}{\left(1-(\theta x)^{k}\right)^{2}} \\
& \times\left(1-\left[1-\left(1-\left(1+\left(\frac{(\theta x)^{k}}{1-(\theta x)^{k}}\right)^{-\alpha}\right)^{-\beta}\right)^{2}\right]^{b}\right)^{-1} .
\end{aligned}
$$
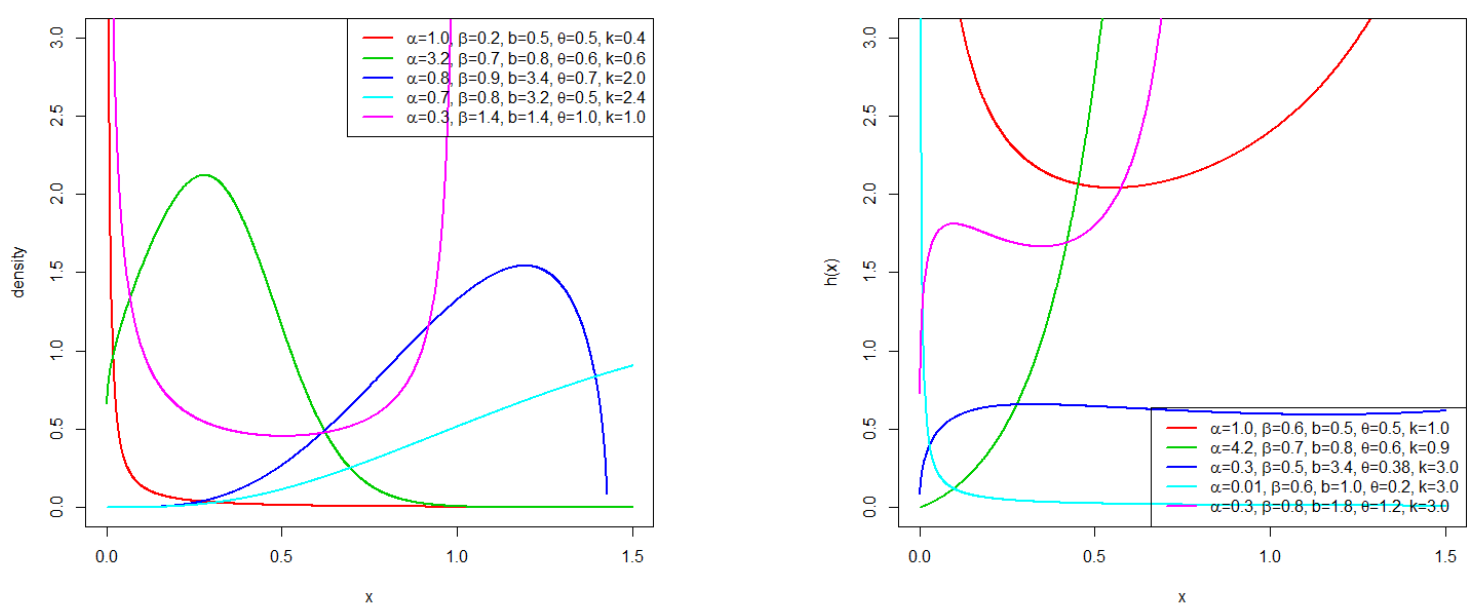

Figure 2. Density and Hazard Rate Function Plots for TL-OBIII-Power Distribution 
Figure 2 shows the plots of pdf and hrf of the TL-OBIII-P distribution, respectively. The pdf can take several shapes including increasing, right-skewed, U-shape and reverse-J shapes. The TL-OBIII-P hrf displays increasing, reverse-J, bathtub, upside-down bathtub followed by bathtub, and upside-down bathtub shapes.

Table 2. Table of Quantile for TL-OBIII-P Distribution

\begin{tabular}{c|ccccc}
\hline \multicolumn{5}{c}{$(\alpha, \beta, b, a, \theta)$} \\
\hline$u$ & $(1.4,1,0.4,1.8,0.3)$ & $(0.6,1.3,2.2,1,1.2)$ & $(0.8,1,2.6,1.7,0.5)$ & $(2,1.0,1.0,2,1.0)$ & $(1,1.8,0.6,2.5,0.5)$ \\
\hline 0.1 & 0.7849 & 0.7447 & 0.1180 & 0.2946 & 0.7221 \\
0.2 & 1.2038 & 1.0361 & 0.1813 & 0.4059 & 0.9034 \\
0.3 & 1.5896 & 1.2875 & 0.2397 & 0.4897 & 1.0497 \\
0.4 & 1.9807 & 1.5323 & 0.2989 & 0.5605 & 1.1866 \\
0.5 & 2.4011 & 1.7882 & 0.3628 & 0.6240 & 1.3261 \\
0.6 & 2.8779 & 2.0726 & 0.4353 & 0.6837 & 1.4782 \\
0.7 & 3.4540 & 2.4114 & 0.5231 & 0.7420 & 1.6568 \\
0.8 & 4.2202 & 2.8583 & 0.6399 & 0.8019 & 1.8902 \\
0.9 & 5.4586 & 3.5807 & 0.8293 & 0.8694 & 2.2648 \\
\hline
\end{tabular}

2.3.3. TL-OBIII-Lomax Distribution Consider Lomax distribution as the baseline distribution with cdf and pdf are given by $G(x ; a, \theta)=1-(1+\theta x)^{-a}$ and $g(x ; a, \theta)=a \theta(1+\theta x)^{-a-1}$, for $a, \theta>0$, and $x>0$. The TL-OBIIILomax (TL-OBIII-L) distribution has cdf and pdf given by

$$
F(x ; \alpha, \beta, b, a, \theta)=\left[1-\left(1-\left(1+\left(\frac{1-(1+\theta x)^{-a}}{(1+\theta x)^{-a}}\right)^{-\alpha}\right)^{-\beta}\right)^{2}\right]^{b}
$$

and

$$
\begin{aligned}
f(x ; \alpha, \beta, b, a, \theta) & =2 \alpha \beta b\left[1-\left(1-\left(1+\left(\frac{1-(1+\theta x)^{-a}}{(1+\theta x)^{-a}}\right)^{-\alpha}\right)^{-\beta}\right)^{2}\right]^{b-1} \\
& \times\left(1-\left(1+\left(\frac{1-(1+\theta x)^{-a}}{(1+\theta x)^{-a}}\right)^{-\alpha}\right)^{-\beta}\right) \frac{a \theta(1+\theta x)^{-a-1}}{\left((1+\theta x)^{-a}\right)^{2}} \\
& \times\left(1+\left(\frac{1-(1+\theta x)^{-a}}{(1+\theta x)^{-a}}\right)^{-\alpha}\right)^{-\beta-1}\left(\frac{1-(1+\theta x)^{-a}}{(1+\theta x)^{-a}}\right)^{-\alpha-1},
\end{aligned}
$$


respectively, for $\alpha, \beta, b, a, \theta>0$. The hrf is given by

$$
\begin{aligned}
h_{F}(x ; \alpha, \beta, b, a, \theta) & =2 \alpha \beta b\left[1-\left(1-\left(1+\left(\frac{1-(1+\theta x)^{-a}}{(1+\theta x)^{-a}}\right)^{-\alpha}\right)^{-\beta}\right)^{2}\right]^{b-1} \\
& \times\left(1-\left(1+\left(\frac{1-(1+\theta x)^{-a}}{(1+\theta x)^{-a}}\right)^{-\alpha}\right)^{-\beta}\right) \frac{a \theta(1+\theta x)^{-a-1}}{\left((1+\theta x)^{-a}\right)^{2}} \\
& \times\left(1+\left(\frac{1-(1+\theta x)^{-a}}{(1+\theta x)^{-a}}\right)^{-\alpha}\right)^{-\beta-1}\left(\frac{1-(1+\theta x)^{-a}}{(1+\theta x)^{-a}}\right)^{-\alpha-1} \\
& \times\left(1-\left[1-\left(1-\left(1+\left(\frac{1-(1+\theta x)^{-a}}{(1+\theta x)^{-a}}\right)^{-\alpha}\right)^{-\beta}\right)^{2}\right]^{b}\right)^{-1}
\end{aligned}
$$
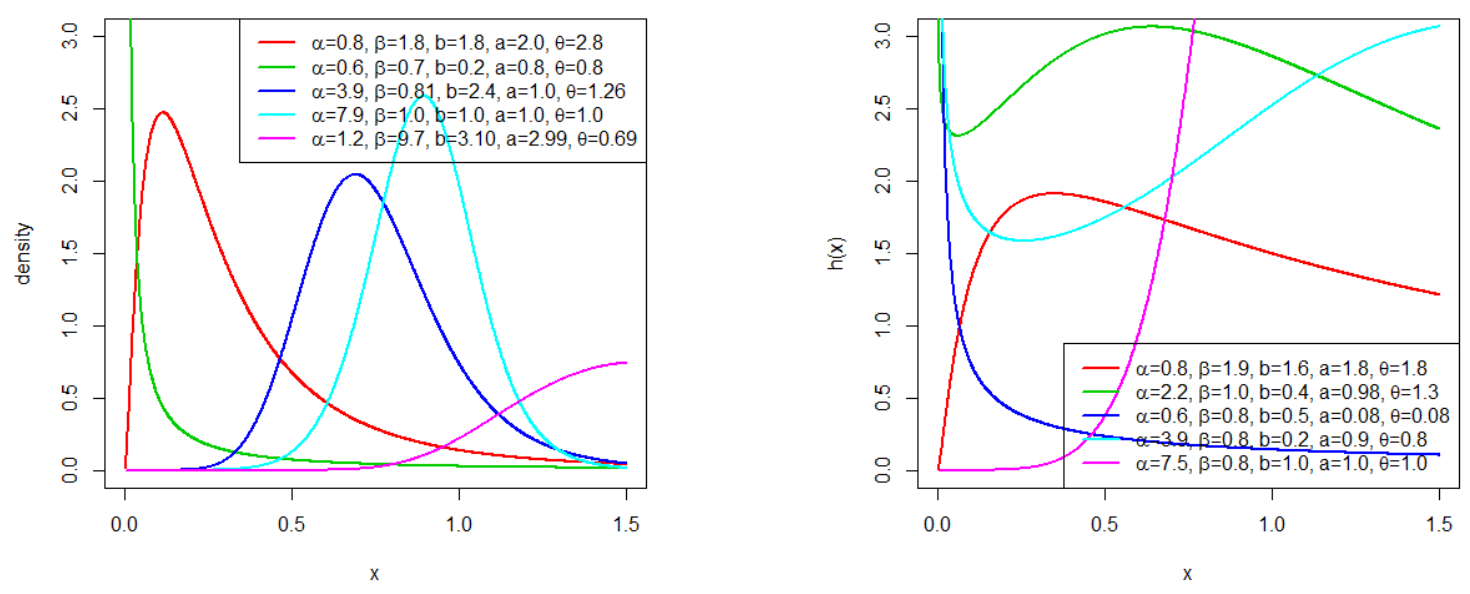

Figure 3. Density and Hazard Rate Function Plots for TL-OBIII-L Distribution

Figure 3 shows the plots of pdf and hrf functions of TL-OBIII-L distribution, respectively. The pdf can take several shapes including right-skewed, left-skewed, almost symmetric, and reverse-J shapes. The TL-OBIII-L hrf displays increasing, decreasing, bathtub, bathtub followed by upside-down bathtub and upside-down bathtub shapes. 
Table 3. Table of Quantile for TL-OBIII-L Distribution

\begin{tabular}{c|ccccc}
\hline \multicolumn{5}{c}{$(\alpha, \beta, b, a, \theta)$} \\
\hline$u$ & $(1.8,0.9,0.8,0.8,1.0)$ & $(2.0,0.8,0.5,0.4,2.0)$ & $(2.5,0.6,0.7,1.5,1.8)$ & $(1.4,1.3,1.9,2.0,2.0)$ & $(0.8,1.7,0.8,0.4,1.9)$ \\
\hline 0.1 & 0.1414 & 0.0448 & 0.0249 & 0.1008 & 0.1205 \\
0.2 & 0.2545 & 0.1167 & 0.0501 & 0.1340 & 0.2808 \\
0.3 & 0.3619 & 0.2034 & 0.0749 & 0.1657 & 0.5182 \\
0.4 & 0.4836 & 0.3169 & 0.0995 & 0.1934 & 0.8791 \\
0.5 & 0.6176 & 0.4601 & 0.1250 & 0.2282 & 1.4738 \\
0.6 & 0.7764 & 0.6440 & 0.1597 & 0.2629 & 2.5450 \\
0.7 & 0.9849 & 0.9101 & 0.1918 & 0.3094 & 4.8028 \\
0.8 & 1.2809 & 1.3367 & 0.2352 & 0.3733 & 10.9256 \\
0.9 & 1.8431 & 2.2512 & 0.3060 & 0.4878 & 40.3184 \\
\hline
\end{tabular}

\subsection{Series Expansion of Density Function}

In this section, we write the pdf of TL-OBIII-G family of distribution as a linear combination of exponentiated-G (E-G) distribution, which will be used for further computations. Applying the following expansions

$$
(1-z)^{k}=\sum_{j=0}^{\infty}(-1)^{j}\left(\begin{array}{l}
k \\
j
\end{array}\right) z^{j} \quad \text { and } \quad(1+z)^{-\beta-1}=\sum_{l=0}^{\infty} \frac{\Gamma((\beta+1)+l)}{\Gamma(\beta+1) l !}(-1)^{l} z^{l}
$$

for $|z|<1$, the pdf of TL-OBIII-G family of distributions can be written as

$$
\begin{aligned}
f(x ; \alpha, \beta, b, \xi) & =2 \alpha \beta b\left[1-\left(1-\left(1+\left(\frac{G(x ; \xi)}{\bar{G}(x ; \xi)}\right)^{-\alpha}\right)^{-\beta}\right]^{2}\right]^{b-1} \\
& \times\left(1-\left(1+\left(\frac{G(x ; \xi)}{\bar{G}(x ; \xi)}\right)^{-\alpha}\right)^{-\beta}\right)\left(1+\left(\frac{G(x ; \xi)}{\bar{G}(x ; \xi)}\right)^{-\alpha}\right)^{-\beta-1} \\
& \times\left(\frac{G(x ; \xi)}{\bar{G}(x ; \xi)}\right)^{-\alpha-1} \frac{g(x ; \xi)}{(\bar{G}(x ; \xi))^{2}} \\
& =2 \alpha \beta b \sum_{j=0}^{\infty}\left(\begin{array}{c}
b-1 \\
j
\end{array}\right)(-1)^{j}\left(1-\left(1+\left(\frac{G(x ; \xi)}{\bar{G}(x ; \xi)}\right)^{-\alpha}\right)^{-\beta}\right)^{2 j+1} \\
& \times\left(1+\left(\frac{G(x ; \xi)}{\bar{G}(x ; \xi)}\right)^{-\alpha}\right)^{-\beta-1}\left(\frac{G(x ; \xi)}{\bar{G}(x ; \xi)}\right)^{-\alpha-1} \frac{g(x ; \xi)}{(\bar{G}(x ; \xi))^{2}} \\
& =2 \alpha \beta b \sum_{j, p=0}^{\infty}\left(\begin{array}{c}
b-1 \\
j
\end{array}\right)\left(\begin{array}{c}
2 j+1 \\
p
\end{array}\right)^{-1)^{j+p}} \\
& \times\left(1+\left(\frac{G(x ; \xi)}{\bar{G}(x ; \xi)}\right)^{-\alpha}\right)^{-\beta(p+1)-1}\left(\frac{G(x ; \xi)}{\bar{G}(x ; \xi)}\right)^{-\alpha-1} \frac{g(x ; \xi)}{(\bar{G}(x ; \xi))^{2}}
\end{aligned}
$$




$$
\begin{aligned}
& =2 \alpha \beta b \sum_{j, p, l=0}^{\infty}\left(\begin{array}{c}
b-1 \\
j
\end{array}\right)\left(\begin{array}{c}
2 j+1 \\
p
\end{array}\right) \frac{\Gamma(\beta(p+1)+l+1)}{\Gamma(\beta(p+1)+1) l !}(-1)^{j+p+l} \\
& \times\left(\frac{G(x ; \xi)}{\bar{G}(x ; \xi)}\right)^{-\alpha(l+1)-1} \frac{g(x ; \xi)}{(\bar{G}(x ; \xi))^{2}} \\
& =2 \alpha \beta b \sum_{j, p, l=0}^{\infty}\left(\begin{array}{c}
b-1 \\
j
\end{array}\right)\left(\begin{array}{c}
2 j+1 \\
p
\end{array}\right) \frac{\Gamma(\beta(p+1)+l+1)}{\Gamma(\beta(p+1)+1) l !}(-1)^{j+p+l} \\
& \times \quad(G(x ; \xi))^{-\alpha(l+1)-1}(\bar{G}(x ; \xi))^{\alpha(l+1)-1} g(x ; \xi) \\
& =2 \alpha \beta b \sum_{j, p, l=0}^{\infty}\left(\begin{array}{c}
b-1 \\
j
\end{array}\right)\left(\begin{array}{c}
2 j+1 \\
p
\end{array}\right) \frac{\Gamma(\beta(p+1)+l+1)}{\Gamma(\beta(p+1)+1) l !}(-1)^{j+p+l} \\
& \times \quad(1-(1-G(x ; \xi)))^{-\alpha(l+1)-1}(\bar{G}(x ; \xi))^{\alpha(l+1)-1} g(x ; \xi) \\
& =2 \alpha \beta b \sum_{j, p, l, k=0}^{\infty}\left(\begin{array}{c}
b-1 \\
j
\end{array}\right)\left(\begin{array}{c}
2 j+1 \\
p
\end{array}\right) \frac{\Gamma(\beta(p+1)+l+1)}{\Gamma(\beta(p+1)+1) l !} \frac{\Gamma(\alpha(l+1)+1+k)}{\Gamma(\alpha(l+1)+1) k !} \\
& \times(-1)^{j+p+l}(\bar{G}(x ; \xi))^{k+\alpha(l+1)-1} g(x ; \xi) \\
& =2 \alpha \beta b \sum_{j, p, l, k, v=0}^{\infty}\left(\begin{array}{c}
b-1 \\
j
\end{array}\right)\left(\begin{array}{c}
2 j+1 \\
p
\end{array}\right) \frac{\Gamma(\beta(p+1)+l+1)}{\Gamma(\beta(p+1)+1) l !} \frac{\Gamma(\alpha(l+1)+1+k)}{\Gamma(\alpha(l+1)+1) k !} \\
& \times(-1)^{j+p+l+k}\left(\begin{array}{c}
k+\alpha(l+1)-1 \\
v
\end{array}\right)(G(x ; \xi))^{v} g(x ; \xi) \\
& =2 \alpha \beta b \sum_{j, p, l, k, v=0}^{\infty}\left(\begin{array}{c}
b-1 \\
j
\end{array}\right)\left(\begin{array}{c}
2 j+1 \\
p
\end{array}\right) \frac{\Gamma(\beta(p+1)+l+1)}{\Gamma(\beta(p+1)+1) l !} \frac{\Gamma(\alpha(l+1)+1+k)}{\Gamma(\alpha(l+1)+1) k !} \\
& \times(-1)^{j+p+l+k}\left(\begin{array}{c}
k+\alpha(l+1)-1 \\
v
\end{array}\right) \frac{v+1}{v+1}(G(x ; \xi))^{v} g(x ; \xi) \\
& =\sum_{v=0}^{\infty} C_{v+1} g_{v+1}(x ; \xi),
\end{aligned}
$$

where $g_{v+1}(x ; \xi)=(v+1)[G(x ; \xi)]^{v} g(x ; \xi)$ is the exponentiated-G $(\mathrm{E}-\mathrm{G})$ pdf with the power parameter $v+1>0$ and parameter vector $\xi$, and

$$
\begin{aligned}
C_{v+1} & =2 \alpha \beta b \sum_{j, p, l, k=0}^{\infty}\left(\begin{array}{c}
b-1 \\
j
\end{array}\right)\left(\begin{array}{c}
2 j+1 \\
p
\end{array}\right) \frac{\Gamma(\beta(p+1)+l+1)}{\Gamma(\beta(p+1)+1) l !} \frac{\Gamma(\alpha(l+1)+1+k)}{\Gamma(\alpha(l+1)+1) k !} \\
& \times(-1)^{j+p+l+k}\left(\begin{array}{c}
k+\alpha(l+1)-1 \\
v
\end{array}\right) \frac{1}{v+1} .
\end{aligned}
$$

Therefore, the pdf of TL-OBIII-G family of distributions can be written as an infinite linear combination of exponentiated-G (E-G) densities. The structural properties of the TL-OBIII-G family of distributions follows from those of E-G distribution. 


\subsection{Moments and Generating Function}

From now onwards, let $Y_{v+1} \sim E-G(v+1)$. The $r^{t h}$ raw moment, $\mu_{r}^{\prime}$ of the TL-OBIII-G family of distributions can be obtained from equation (9) as

$$
\begin{aligned}
\mu_{r}^{\prime}=E\left(X^{r}\right) & =\int_{-\infty}^{\infty} x^{r} f(x) d x \\
& =\sum_{v=0}^{\infty} C_{v+1} E\left(Y_{v+1}^{r}\right) .
\end{aligned}
$$

The moment generating function (MGF) $M_{X}(t)=E\left(e^{t X}\right)$ is given by:

$$
M_{X}(t)=\sum_{v=0}^{\infty} C_{v+1} M_{v+1}(t),
$$

where $M_{v+1}(t)$ is the mgf of $Y_{v+1}$ and $C_{v+1}$ is given by equation (10).

Tables 4, 5 and 6 presents the first six moments, standard deviation (SD), coefficient of variation (CV), coefficient of skewness (CS) and coefficient of kurtosis (CK) for selected parameter values of some special cases of the TLOBIII-G family of distributions.

Table 4. Table of Moments for Selected Parameters for TL-OBIII-LLoG Distribution

\begin{tabular}{c|ccccc}
\hline \multicolumn{5}{c}{$(\alpha, \beta, b, \lambda)$} \\
\hline & $(0.4,0.5,0.2,0.5)$ & $(0.9,1.2,1.0,0.6)$ & $(0.3,2.2,1.0,0.2)$ & $(3.0,2.0,0.5,0.6)$ & $(0.8,2.0,1.2,0.5)$ \\
\hline $\mathrm{E}(X)$ & 0.0053 & 0.1599 & 0.0220 & 0.3490 & 0.1208 \\
$\mathrm{E}\left(X^{2}\right)$ & 0.0024 & 0.0830 & 0.0111 & 0.2261 & 0.0689 \\
$\mathrm{E}\left(X^{3}\right)$ & 0.0015 & 0.0552 & 0.0074 & 0.1637 & 0.0478 \\
$\mathrm{E}\left(X^{4}\right)$ & 0.0011 & 0.0412 & 0.0055 & 0.1270 & 0.0365 \\
$\mathrm{E}\left(X^{5}\right)$ & 0.0009 & 0.0328 & 0.0044 & 0.1032 & 0.0295 \\
$\mathrm{E}\left(X^{6}\right)$ & 0.0007 & 0.0272 & 0.0037 & 0.0866 & 0.0247 \\
$\mathrm{SD}$ & 0.0006 & 0.0232 & 0.0032 & 0.0745 & 0.0213 \\
$\mathrm{CV}$ & 0.0005 & 0.0203 & 0.0028 & 0.0653 & 0.0187 \\
$\mathrm{CS}$ & 0.0005 & 0.0180 & 0.0024 & 0.0581 & 0.0166 \\
$\mathrm{CK}$ & 0.0004 & 0.0162 & 0.0022 & 0.0522 & 0.0150 \\
\hline
\end{tabular}

Table 5. Table of Moments for Selected Parameters for TL-OBIII-Lomax Distribution

\begin{tabular}{c|ccccc}
\hline \multicolumn{5}{c}{$(\alpha, \beta, b, a, \theta)$} \\
\hline & $(0.4,0.5,0.2,0.5)$ & $(0.9,1.2,1.0,0.6)$ & $(0.3,2.2,1.0,0.2)$ & $(3.0,2.0,0.5,0.6)$ & $(0.8,2.0,1.2,0.5)$ \\
\hline $\mathrm{E}(X)$ & 0.2291 & 0.0822 & 0.07376 & 0.0170 & 0.1920 \\
$\mathrm{E}\left(X^{2}\right)$ & 0.1075 & 0.0396 & 0.0344 & 0.0036 & 0.0480 \\
$\mathrm{E}\left(X^{3}\right)$ & 0.0662 & 0.0257 & 0.0222 & 0.0015 & 0.0155 \\
$\mathrm{E}\left(X^{4}\right)$ & 0.0469 & 0.0189 & 0.0163 & 0.0009 & 0.0064 \\
$\mathrm{E}\left(X^{5}\right)$ & 0.0360 & 0.0150 & 0.0129 & 0.0006 & 0.0032 \\
$\mathrm{E}\left(X^{6}\right)$ & 0.0291 & 0.0124 & 0.0107 & 0.0004 & 0.0019 \\
$\mathrm{SD}$ & 0.0244 & 0.0105 & 0.0091 & 0.0003 & 0.0013 \\
$\mathrm{CV}$ & 0.0210 & 0.0092 & 0.0079 & 0.0003 & 0.0010 \\
$\mathrm{CS}$ & 0.0184 & 0.0081 & 0.0070 & 0.0002 & 0.0007 \\
$\mathrm{CK}$ & 0.0164 & 0.0073 & 0.0063 & 0.0002 & 0.0006 \\
\hline
\end{tabular}


Table 6. Table of Moments for Selected Parameters for TL-OBIII-Power Distribution

\begin{tabular}{c|ccccc}
\hline \multicolumn{7}{c}{$(\alpha, \beta, b, a, \theta)$} & & & \\
\hline & $(0.4,0.5,0.2,0.5,0.2)$ & $(0.9,1.2,1.0,0.6,2.0)$ & $(0.3,2.2,1.0,0.2,3.0)$ & $(0.5,2.0,0.5,0.6,0.8)$ & $(0.5,2.0,1.2,0.5,0.2)$ \\
\hline $\mathrm{E}(X)$ & 0.0046 & 0.3398 & 0.0323 & 0.1908 & 0.1570 \\
$\mathrm{E}\left(X^{2}\right)$ & 0.0025 & 0.2507 & 0.0233 & 0.1119 & 0.0931 \\
$\mathrm{E}\left(X^{3}\right)$ & 0.0017 & 0.1979 & 0.0182 & 0.0799 & 0.0667 \\
$\mathrm{E}\left(X^{4}\right)$ & 0.0013 & 0.1632 & 0.0149 & 0.0624 & 0.0522 \\
$\mathrm{E}\left(X^{5}\right)$ & 0.0011 & 0.1387 & 0.0127 & 0.0513 & 0.0429 \\
$\mathrm{E}\left(X^{6}\right)$ & 0.0009 & 0.1205 & 0.0109 & 0.0436 & 0.0364 \\
$\mathrm{SD}$ & 0.0008 & 0.1065 & 0.0096 & 0.0379 & 0.0316 \\
$\mathrm{CV}$ & 0.0007 & 0.0954 & 0.0087 & 0.0335 & 0.0279 \\
$\mathrm{CS}$ & 0.0006 & 0.0863 & 0.0078 & 0.0301 & 0.0251 \\
$\mathrm{CK}$ & 0.0005 & 0.0788 & 0.0072 & 0.0273 & 0.0228 \\
\hline
\end{tabular}

\subsection{Incomplete Moments}

When it comes to computing Bonferroni and Lorenz curves, incomplete moments are useful. The $s^{\text {th }}$ incomplete moment of $X$ denoted by $\eta_{s}(t)$ is given by

$$
\eta_{s}(t)=\int_{-\infty}^{t} x^{s} f(x) d x
$$

Using equation (9), we obtain

$$
\eta_{s}(t)=\sum_{v=0}^{\infty} C_{v+1} \int_{-\infty}^{t} x^{s} g_{v+1}(x ; \xi) d x .
$$

By setting $s=1$ in equation (11), we obtain the first incomplete moment of the TL-OBIII-G family of distributions.

\subsection{Rényi Entropy}

Rényi entropy (Rényi [31]) is an extension of Shannon entropy. Rényi entropy is defined to be

$$
I_{R}(v)=\frac{1}{1-v} \log \left(\int_{0}^{\infty}[f(x ; \alpha, \beta, b, \xi)]^{v} d x\right), v \neq 1, v>0 .
$$

Rényi entropy tends to Shannon entropy as $v \rightarrow 1$. Note that

$$
\begin{aligned}
{[f(x ; \alpha, \beta, b, \xi)]^{v} } & =(2 \alpha \beta b)^{v}\left[1-\left(1-\left(1+\left(\frac{G(x ; \xi)}{\bar{G}(x ; \xi)}\right)^{-\alpha}\right)^{-\beta}\right)^{2}\right]^{v(b-1)} \\
& \times\left(1-\left(1+\left(\frac{G(x ; \xi)}{\bar{G}(x ; \xi)}\right)^{-\alpha}\right)^{-\beta}\right)^{v}\left(1+\left(\frac{G(x ; \xi)}{\bar{G}(x ; \xi)}\right)^{-\alpha}\right)^{v(-\beta-1)} \\
& \times\left(\frac{G(x ; \xi)}{\bar{G}(x ; \xi)}\right)^{v(-\alpha-1)} \frac{g(x ; \xi)^{v}}{(\bar{G}(x ; \xi))^{2 v}}
\end{aligned}
$$

Using the fact that

$$
\begin{aligned}
{\left[1-\left(1-\left(1+\left(\frac{G(x ; \xi)}{\bar{G}(x ; \xi)}\right)^{-\alpha}\right)^{-\beta}\right)^{2}\right]^{v(b-1)} } & =\sum_{j=0}^{\infty}\left(\begin{array}{c}
v(b-1) \\
j
\end{array}\right)(-1)^{j} \\
& \times\left(1-\left(1+\left(\frac{G(x ; \xi)}{\bar{G}(x ; \xi)}\right)^{-\alpha}\right)^{-\beta}\right)^{2 j}
\end{aligned}
$$


we write

$$
\begin{aligned}
{[f(x ; \alpha, \beta, b, \xi)]^{v} } & =(2 \alpha \beta b)^{v} \sum_{j=0}^{\infty}\left(\begin{array}{c}
v(b-1) \\
j
\end{array}\right)(-1)^{j}\left(1+\left(\frac{G(x ; \xi)}{\bar{G}(x ; \xi)}\right)^{-\alpha}\right)^{v(-\beta-1)} \\
& \times\left(1-\left(1+\left(\frac{G(x ; \xi)}{\bar{G}(x ; \xi)}\right)^{-\alpha}\right)^{-\beta}\right)^{2 j+v}\left(\frac{G(x ; \xi)}{\bar{G}(x ; \xi)}\right)^{v(-\alpha-1)} \\
& \times \frac{g(x ; \xi)^{v}}{(\bar{G}(x ; \xi))^{2 v}}
\end{aligned}
$$

Using the expansion

$$
\begin{aligned}
\left(1-\left(1+\left(\frac{G(x ; \xi)}{\bar{G}(x ; \xi)}\right)^{-\alpha}\right)^{-\beta}\right)^{2 j+v} & =\sum_{p=0}^{\infty}\left(\begin{array}{c}
2 j+v \\
p
\end{array}\right)(-1)^{p} \\
& \times\left(1+\left(\frac{G(x ; \xi)}{\bar{G}(x ; \xi)}\right)^{-\alpha}\right)^{-\beta p}
\end{aligned}
$$

we write

$$
\begin{aligned}
{[f(x ; \alpha, \beta, b, \xi)]^{v} } & =(2 \alpha \beta b)^{v} \sum_{j, p=0}^{\infty}\left(\begin{array}{c}
v(b-1) \\
j
\end{array}\right)\left(\begin{array}{c}
2 j+v \\
p
\end{array}\right)\left(\frac{G(x ; \xi)}{\bar{G}(x ; \xi)}\right)^{v(-\alpha-1)} \\
& \times(-1)^{j+p}\left(1+\left(\frac{G(x ; \xi)}{\bar{G}(x ; \xi)}\right)^{-\alpha}\right)^{-\beta(v+p)-v} \frac{g(x ; \xi)^{v}}{(\bar{G}(x ; \xi))^{2 v}}
\end{aligned}
$$

\section{Considering}

$$
\left(1+\left(\frac{G(x ; \xi)}{\bar{G}(x ; \xi)}\right)^{-\alpha}\right)^{-\beta(v+p)-v}=\sum_{l=0}^{\infty}(-1)^{l} \frac{\Gamma(\beta(v+p)+v+l)}{\Gamma(\beta(v+p)+v) l !}\left(\frac{G(x ; \xi)}{\bar{G}(x ; \xi)}\right)^{-\alpha l}
$$


we have

$$
\begin{aligned}
{[f(x ; \alpha, \beta, b, \xi)]^{v} } & =(2 \alpha \beta b)^{v} \sum_{j, p, l=0}^{\infty}\left(\begin{array}{c}
v(b-1) \\
j
\end{array}\right)\left(\begin{array}{c}
2 j+v \\
p
\end{array}\right)(-1)^{j+p+l} g(x ; \xi)^{v} \\
& \times \frac{\Gamma(\beta(v+p)+v+l)}{\Gamma(\beta(v+p)+v) l !}(G(x ; \xi))^{-\alpha(v+l)-v}(\bar{G}(x ; \xi))^{\alpha(v+l)-v} \\
& =(2 \alpha \beta b)^{v} \sum_{j, p, l=0}^{\infty}\left(\begin{array}{c}
v(b-1) \\
j
\end{array}\right)\left(\begin{array}{c}
2 j+v \\
p
\end{array}\right)(-1)^{j+p+l} g(x ; \xi)^{v} \\
& \times \frac{\Gamma(\beta(v+p)+v+l)}{\Gamma(\beta(v+p)+v) l !}(1-(1-G(x ; \xi)))^{-\alpha(v+l)-v}(\bar{G}(x ; \xi))^{\alpha(v+l)-v} \\
& =(2 \alpha \beta b)^{v} \sum_{j, p, l, k=0}^{\infty}\left(\begin{array}{c}
v(b-1) \\
j
\end{array}\right)\left(\begin{array}{c}
2 j+v \\
p
\end{array}\right)(-1)^{j+p+l} g(x ; \xi)^{v} \\
& \times \frac{\Gamma(\alpha(v+l)+v+k)}{\Gamma(\alpha(v+l)+v) k !} \frac{\Gamma(\beta(v+p)+v+l)}{\Gamma(\beta(v+p)+v) l !}(\bar{G}(x ; \xi))^{k+\alpha(v+l)-v} \\
& =(2 \alpha \beta b)^{v} \sum_{j, p, l, k, q=0}^{\infty}\left(\begin{array}{c}
v(b-1) \\
j
\end{array}\right)\left(\begin{array}{c}
2 j+v \\
p
\end{array}\right)\left(\begin{array}{c}
k+\alpha(v+l)-v \\
q
\end{array}\right) \\
& \times(-1)^{j+p+l} \frac{\Gamma(\alpha(v+l)+v+k)}{\Gamma(\alpha(v+l)+v) k !} \frac{\Gamma(\beta+p)+v+l)}{\Gamma(\beta(v+p)+v) l !}(G(x ; \xi))^{q} g(x ; \xi)^{v} .
\end{aligned}
$$

Consequently, Rényi entropy for the TL-OBIII-G family of distributions is given by

$$
\begin{aligned}
I_{R}(v) & =\frac{1}{1-v} \log \left[(2 \alpha \beta b)^{v} \sum_{j, p, l, k, q=0}^{\infty}\left(\begin{array}{c}
v(b-1) \\
j
\end{array}\right)\left(\begin{array}{c}
2 j+v \\
p
\end{array}\right)\left(\begin{array}{c}
k+\alpha(v+l)-v \\
q
\end{array}\right)\right. \\
& \times(-1)^{j+p+l} \frac{\Gamma(\alpha(v+l)+v+k)}{\Gamma(\alpha(v+l)+v) k !} \frac{\Gamma(\beta(v+p)+v+l)}{\Gamma(\beta(v+p)+v) l !} \frac{1}{\left[\frac{q}{v}+1\right]^{v}} \\
& \left.\times \int_{0}^{\infty}\left(\left[\frac{q}{v}+1\right](G(x ; \xi))^{\frac{q}{v}} g(x ; \xi)\right) d x\right] \\
& =\frac{1}{1-v} \log \left[\sum_{j, p, l, k, q=0}^{\infty} w_{j, p, l, k, q} \exp \left((1-v) I_{R E G}\right)\right],
\end{aligned}
$$

for $v>0, v \neq 1$, where

$I_{R E G}=\frac{1}{1-v} \log \left[\int_{0}^{\infty}\left(\left[\frac{q}{v}+1\right](G(x ; \xi))^{\frac{q}{v}} g(x ; \xi)\right)^{v} d x\right]$ is the Rényi entropy of E-G distribution with power parameter $\frac{q}{v}+1$, and

$$
\begin{aligned}
w_{j, p, l, k, q} & =(2 \alpha \beta b)^{v} \sum_{j, p, l, k, q=0}^{\infty}\left(\begin{array}{c}
v(b-1) \\
j
\end{array}\right)\left(\begin{array}{c}
2 j+v \\
p
\end{array}\right)\left(\begin{array}{c}
k+\alpha(v+l)-v \\
q
\end{array}\right) \\
& \times(-1)^{j+p+l} \frac{\Gamma(\alpha(v+l)+v+k)}{\Gamma(\alpha(v+l)+v) k !} \frac{\Gamma(\beta(v+p)+v+l)}{\Gamma(\beta(v+p)+v) l !} \frac{1}{\left[\frac{q}{v}+1\right]^{v}} .
\end{aligned}
$$

\subsection{Order Statistics}

Let $X_{1}, X_{2}, \ldots, X_{n}$ be a simple random sample from the TL-OBIII-G family of distributions. Using the binomial expansion 
$(1-F(x))^{n-i}=\sum_{m=0}^{n-i}\left(\begin{array}{c}n-i \\ m\end{array}\right)(-1)^{m}[F(x)]^{m}$, the pdf of the $i^{t h}$ order statistic can be expressed as

$$
\begin{aligned}
f_{i: n}(x) & =\frac{n ! f(x)}{(i-1) !(n-i) !}[F(x)]^{i-1}[1-F(x)]^{n-i} \\
& =\frac{n ! f(x)}{(i-1) !(n-i) !} \sum_{m=0}^{n-i}(-1)^{m}\left(\begin{array}{c}
n-i \\
m
\end{array}\right)[F(x)]^{m+i-1}
\end{aligned}
$$

Based on equations (3) and (4), we can write

$$
\begin{aligned}
& f(x) F(x)^{m+i-1}=2 \alpha \beta b\left[1-\left(1-\left(1+\left(\frac{G(x ; \xi)}{\bar{G}(x ; \xi)}\right)^{-\alpha}\right)^{-\beta}\right)^{2}\right]^{b(m+i)-1} \\
& \times\left(1-\left(1+\left(\frac{G(x ; \xi)}{\bar{G}(x ; \xi)}\right)^{-\alpha}\right)^{-\beta}\right)\left(1+\left(\frac{G(x ; \xi)}{\bar{G}(x ; \xi)}\right)^{-\alpha}\right)^{-\beta-1} \\
& \times\left(\frac{G(x ; \xi)}{\bar{G}(x ; \xi)}\right)^{-\alpha-1} \frac{g(x ; \xi)}{(\bar{G}(x ; \xi))^{2}} \\
& =\sum_{j=0}^{\infty}\left(\begin{array}{c}
b(m+i)-1 \\
j
\end{array}\right)(-1)^{j}\left(1-\left(1+\left(\frac{G(x ; \xi)}{\bar{G}(x ; \xi)}\right)^{-\alpha}\right)^{-\beta}\right)^{2 j+1} \\
& \times 2 \alpha \beta b\left(1+\left(\frac{G(x ; \xi)}{\bar{G}(x ; \xi)}\right)^{-\alpha}\right)^{-\beta-1}\left(\frac{G(x ; \xi)}{\bar{G}(x ; \xi)}\right)^{-\alpha-1} \frac{g(x ; \xi)}{(\bar{G}(x ; \xi))^{2}} \\
& =2 \alpha \beta b \sum_{j, p=0}^{\infty}\left(\begin{array}{c}
b(m+i)-1 \\
j
\end{array}\right)\left(\begin{array}{c}
2 j+1 \\
p
\end{array}\right)(-1)^{j+p} \\
& \times\left(1+\left(\frac{G(x ; \xi)}{\bar{G}(x ; \xi)}\right)^{-\alpha}\right)^{-\beta(p+1)-1}\left(\frac{G(x ; \xi)}{\bar{G}(x ; \xi)}\right)^{-\alpha-1} \frac{g(x ; \xi)}{(\bar{G}(x ; \xi))^{2}} \\
& =2 \alpha \beta b \sum_{j, p, l=0}^{\infty}\left(\begin{array}{c}
b(m+i)-1 \\
j
\end{array}\right)\left(\begin{array}{c}
2 j+1 \\
p
\end{array}\right) \frac{\Gamma(\beta(p+1)+l+1)}{\Gamma(\beta(p+1)+1) l !} \\
& \times(-1)^{j+p+l}\left(\frac{G(x ; \xi)}{\bar{G}(x ; \xi)}\right)^{-\alpha(l+1)-1} \frac{g(x ; \xi)}{(\bar{G}(x ; \xi))^{2}} \\
& =2 \alpha \beta b \sum_{j, p, l=0}^{\infty}\left(\begin{array}{c}
b(m+i)-1 \\
j
\end{array}\right)\left(\begin{array}{c}
2 j+1 \\
p
\end{array}\right) \frac{\Gamma(\beta(p+1)+l+1)}{\Gamma(\beta(p+1)+1) l !} \\
& \times \quad(-1)^{j+p+l}(G(x ; \xi))^{-\alpha(l+1)-1}(\bar{G}(x ; \xi))^{\alpha(l+1)-1} g(x ; \xi) \\
& =2 \alpha \beta b \sum_{j, p, l=0}^{\infty}\left(\begin{array}{c}
b(m+i)-1 \\
j
\end{array}\right)\left(\begin{array}{c}
2 j+1 \\
p
\end{array}\right) \frac{\Gamma(\beta(p+1)+l+1)}{\Gamma(\beta(p+1)+1) l !}(-1)^{j+p+l} \\
& \times(1-(1-G(x ; \xi)))^{-\alpha(l+1)-1}(\bar{G}(x ; \xi))^{\alpha(l+1)-1} g(x ; \xi)
\end{aligned}
$$




$$
\begin{aligned}
& =2 \alpha \beta b \sum_{j, p, l, k=0}^{\infty}\left(\begin{array}{c}
b(m+i)-1 \\
j
\end{array}\right)\left(\begin{array}{c}
2 j+1 \\
p
\end{array}\right) \frac{\Gamma(\beta(p+1)+l+1)}{\Gamma(\beta(p+1)+1) l !} \frac{\Gamma(\alpha(l+1)+1+k)}{\Gamma(\alpha(l+1)+1) k !} \\
& \times \quad(-1)^{j+p+l}(\bar{G}(x ; \xi))^{k+\alpha(l+1)-1} g(x ; \xi) \\
& =2 \alpha \beta b \sum_{j, p, l, k, v=0}^{\infty}\left(\begin{array}{c}
b(m+i)-1 \\
j
\end{array}\right)\left(\begin{array}{c}
2 j+1 \\
p
\end{array}\right) \frac{\Gamma(\beta(p+1)+l+1)}{\Gamma(\beta(p+1)+1) l !} \frac{\Gamma(\alpha(l+1)+1+k)}{\Gamma(\alpha(l+1)+1) k !} \\
& \times \quad(-1)^{j+p+l+k}\left(\begin{array}{c}
k+\alpha(l+1)-1 \\
v
\end{array}\right) \frac{v+1}{v+1}(G(x ; \xi))^{v} g(x ; \xi) \\
& =\sum_{v=0}^{\infty} b_{v+1}^{*} g_{v+1}(x ; \xi),
\end{aligned}
$$

where

$$
\begin{aligned}
b_{v+1}^{*} & =2 \alpha \beta b \sum_{j, p, l, k, v=0}^{\infty}\left(\begin{array}{c}
b(m+i)-1 \\
j
\end{array}\right)\left(\begin{array}{c}
2 j+1 \\
p
\end{array}\right) \frac{\Gamma(\beta(p+1)+l+1)}{\Gamma(\beta(p+1)+1) l !} \frac{\Gamma(\alpha(l+1)+1+k)}{\Gamma(\alpha(l+1)+1) k !} \\
& \times(-1)^{j+p+l+k}\left(\begin{array}{c}
k+\alpha(l+1)-1 \\
v
\end{array}\right) \frac{1}{v+1} .
\end{aligned}
$$

Substituting equation (15) into equation (14), we obtain

$$
f_{i: n}(x)=\frac{n !}{(i-1) !(n-i) !} \sum_{v=0}^{\infty} \sum_{m=0}^{n-i}(-1)^{m}\left(\begin{array}{c}
n-i \\
m
\end{array}\right) b_{v+1}^{*} g_{v+1}(x ; \xi)
$$

where $g_{v+1}(x ; \xi)=(v+1)[G(x ; \xi)]^{v} g(x ; \xi)$ is the exponentiated-G $(\mathrm{E}-\mathrm{G})$ pdf with the power parameter $v+1>0$ and parameter vector $\xi$. Thus, the density function of the TL-OBIII-G order statistics is a linear combination of E-G densities.

\subsection{Probability Weighted Moments (PWMs)}

The $(p, r)^{t h}$ PWMs of X with TL-OBIII-G distribution denoted $M_{p, r}$ is given by

$$
M_{p, r}=E\left(X^{p}(F(X))^{r}\right)=\int_{-\infty}^{\infty} x^{p}(F(x))^{r} f(x) d x
$$


Using equations (3) and (4), we can write

$$
\begin{aligned}
& f(x)(F(x))^{r}=2 \alpha \beta b\left[1-\left(1-\left(1+\left(\frac{G(x ; \xi)}{\bar{G}(x ; \xi)}\right)^{-\alpha}\right)^{-\beta}\right)^{2}\right]^{b(r+1)-1} \\
& \times\left(1-\left(1+\left(\frac{G(x ; \xi)}{\bar{G}(x ; \xi)}\right)^{-\alpha}\right)^{-\beta}\right)\left(1+\left(\frac{G(x ; \xi)}{\bar{G}(x ; \xi)}\right)^{-\alpha}\right)^{-\beta-1} \\
& \times\left(\frac{G(x ; \xi)}{\bar{G}(x ; \xi)}\right)^{-\alpha-1} \frac{g(x ; \xi)}{(\bar{G}(x ; \xi))^{2}} \\
& =2 \alpha \beta b \sum_{j=0}^{\infty}\left(\begin{array}{c}
b(r+1)-1 \\
j
\end{array}\right)(-1)^{j}\left(1-\left(1+\left(\frac{G(x ; \xi)}{\bar{G}(x ; \xi)}\right)^{-\alpha}\right)^{-\beta}\right)^{2 j+1} \\
& \times\left(1+\left(\frac{G(x ; \xi)}{\bar{G}(x ; \xi)}\right)^{-\alpha}\right)^{-\beta-1}\left(\frac{G(x ; \xi)}{\bar{G}(x ; \xi)}\right)^{-\alpha-1} \frac{g(x ; \xi)}{(\bar{G}(x ; \xi))^{2}} \\
& =2 \alpha \beta b \sum_{j, p=0}^{\infty}\left(\begin{array}{c}
b(r+1)-1 \\
j
\end{array}\right)\left(\begin{array}{c}
2 j+1 \\
p
\end{array}\right)(-1)^{j+p} \\
& \times\left(1+\left(\frac{G(x ; \xi)}{\bar{G}(x ; \xi)}\right)^{-\alpha}\right)^{-\beta(p+1)-1}\left(\frac{G(x ; \xi)}{\bar{G}(x ; \xi)}\right)^{-\alpha-1} \frac{g(x ; \xi)}{(\bar{G}(x ; \xi))^{2}} \\
& =2 \alpha \beta b \sum_{j, p, l=0}^{\infty}\left(\begin{array}{c}
b(r+1)-1 \\
j
\end{array}\right)\left(\begin{array}{c}
2 j+1 \\
p
\end{array}\right) \frac{\Gamma(\beta(p+1)+l+1)}{\Gamma(\beta(p+1)+1) l !}(-1)^{j+p+l} \\
& \times\left(\frac{G(x ; \xi)}{\bar{G}(x ; \xi)}\right)^{-\alpha(l+1)-1} \frac{g(x ; \xi)}{(\bar{G}(x ; \xi))^{2}} \\
& =2 \alpha \beta b \sum_{j, p, l=0}^{\infty}\left(\begin{array}{c}
b(r+1)-1 \\
j
\end{array}\right)\left(\begin{array}{c}
2 j+1 \\
p
\end{array}\right) \frac{\Gamma(\beta(p+1)+l+1)}{\Gamma(\beta(p+1)+1) l !}(-1)^{j+p+l} \\
& \times \quad(G(x ; \xi))^{-\alpha(l+1)-1}(\bar{G}(x ; \xi))^{\alpha(l+1)-1} g(x ; \xi) \\
& =2 \alpha \beta b \sum_{j, p, l=0}^{\infty}\left(\begin{array}{c}
b(r+1)-1 \\
j
\end{array}\right)\left(\begin{array}{c}
2 j+1 \\
p
\end{array}\right) \frac{\Gamma(\beta(p+1)+l+1)}{\Gamma(\beta(p+1)+1) l !}(-1)^{j+p+l} \\
& \times \quad(1-(1-G(x ; \xi)))^{-\alpha(l+1)-1}(\bar{G}(x ; \xi))^{\alpha(l+1)-1} g(x ; \xi) \\
& =2 \alpha \beta b \sum_{j, p, l, k=0}^{\infty}\left(\begin{array}{c}
b(r+1)-1 \\
j
\end{array}\right)\left(\begin{array}{c}
2 j+1 \\
p
\end{array}\right) \frac{\Gamma(\beta(p+1)+l+1)}{\Gamma(\beta(p+1)+1) l !} \frac{\Gamma(\alpha(l+1)+1+k)}{\Gamma(\alpha(l+1)+1) k !} \\
& \times \quad(-1)^{j+p+l}(\bar{G}(x ; \xi))^{k+\alpha(l+1)-1} g(x ; \xi) \\
& =2 \alpha \beta b \sum_{j, p, l, k, v=0}^{\infty}\left(\begin{array}{c}
b(r+1)-1 \\
j
\end{array}\right)\left(\begin{array}{c}
2 j+1 \\
p
\end{array}\right) \frac{\Gamma(\beta(p+1)+l+1)}{\Gamma(\beta(p+1)+1) l !} \frac{\Gamma(\alpha(l+1)+1+k)}{\Gamma(\alpha(l+1)+1) k !} \\
& \times(-1)^{j+p+l+k}\left(\begin{array}{c}
k+\alpha(l+1)-1 \\
v
\end{array}\right) \frac{v+1}{v+1}(G(x ; \xi))^{v} g(x ; \xi) \\
& =\sum_{v=0}^{\infty} C_{v+1}^{*} g_{v+1}(x ; \xi),
\end{aligned}
$$


where $g_{v+1}(x ; \xi)=(v+1)[G(x ; \xi)]^{v} g(x ; \xi)$ is the exponentiated-G $(\mathrm{E}-\mathrm{G})$ pdf with the power parameter $v+1>0$ and parameter vector $\xi$, and

$$
\begin{aligned}
C_{v+1}^{*} & =2 \alpha \beta b \sum_{j, p, l, k=0}^{\infty}\left(\begin{array}{c}
b(r+1)-1 \\
j
\end{array}\right)\left(\begin{array}{c}
2 j+1 \\
p
\end{array}\right) \frac{\Gamma(\beta(p+1)+l+1)}{\Gamma(\beta(p+1)+1) l !} \frac{\Gamma(\alpha(l+1)+1+k)}{\Gamma(\alpha(l+1)+1) k !} \\
& \times(-1)^{j+p+l+k}\left(\begin{array}{c}
k+\alpha(l+1)-1 \\
v
\end{array}\right) \frac{1}{v+1} .
\end{aligned}
$$

Consequently, the PWMs of the TL-OBIII-G family of distributions can be written as

$$
M_{p, r}=\int_{-\infty}^{\infty} x^{p} \sum_{v=0}^{\infty} C_{v+1}^{*} g_{v+1}(x ; \xi) d x=\sum_{v=0}^{\infty} C_{v+1}^{*} \int_{-\infty}^{\infty} x^{p} g_{v+1}(x ; \xi) d x
$$

which shows that the $(p, r)^{t h}$ PWMs of TL-OBIII-G family of distributions can be obtained from the moments of the E-G distribution.

\section{Maximum Likelihood Estimation}

The maximum likelihood estimation (MLE) technique is the commonly used method of parameter estimation among others in the literature. Here, we employ the MLE to estimate model parameters of TL-OBIII-G family of distributions. Let $x_{1}, x_{2}, \ldots \ldots, x_{n}$ be a random sample from TL-OBIII-G family of distributions with the parameter vector $\boldsymbol{\Delta}=(\alpha, \beta, b, \xi)^{T}$, then the log-likelihood function $\ell_{n}=\ell_{n}(\boldsymbol{\Delta})$ is given by

$$
\begin{aligned}
\ell_{n}(\boldsymbol{\Delta}) & =n \ln (2 \alpha \beta b)+(b-1) \sum_{i=1}^{n} \ln \left[1-\left(1-\left(1+\left(\frac{G(x ; \xi)}{\bar{G}(x ; \xi)}\right)^{-\alpha}\right)^{-\beta}\right)^{2}\right] \\
& +\sum_{i=1}^{n} \ln \left(1-\left(1+\left(\frac{G(x ; \xi)}{\bar{G}(x ; \xi)}\right)^{-\alpha}\right)^{-\beta}\right)+(-\alpha-1) \sum_{i=1}^{n} \ln \left(\frac{G(x ; \xi)}{\bar{G}(x ; \xi)}\right) \\
& +(-\beta-1) \sum_{i=1}^{n} \ln \left(1+\left(\frac{G(x ; \xi)}{\bar{G}(x ; \xi)}\right)^{-\alpha}\right)+\sum_{i=1}^{n} \ln g(x ; \xi)-2 \sum_{i=1}^{n} \ln (\bar{G}(x ; \xi)) .
\end{aligned}
$$


Elements of the score vector $U(\boldsymbol{\Delta})=\left(\frac{\partial \ell_{n}}{\partial \alpha}, \frac{\partial \ell_{n}}{\partial \beta}, \frac{\partial \ell_{n}}{\partial b}, \frac{\partial \ell_{n}}{\partial \xi_{k}}\right)$ can be readily obtained. Taking derivative of the loglikelihood function with respect to each component of the parameter vector $\alpha, \beta, b$, and $\xi$, we obtain

$$
\begin{aligned}
& \frac{\partial \ell_{n}}{\partial \alpha}=\frac{n}{\alpha}-\sum_{i=1}^{n} \ln \left(\frac{G(x ; \xi)}{\bar{G}(x ; \xi)}\right)+(-\beta-1) \sum_{i=1}^{n} \frac{\left(\frac{G(x ; \xi)}{\overline{G(x ; \xi)}}\right)^{-\alpha} \ln \left(\frac{G(x ; \xi)}{\bar{G}(x ; \xi)}\right)}{\left(1+\left(\frac{G(x ; \xi)}{\overline{G(x ; \xi)}}\right)^{-\alpha}\right)} \\
& -\left((b-1) \sum_{i=1}^{n} \frac{2 \beta\left(1-\left(1+\left(\frac{G(x ; \xi)}{\overline{G(x ; \xi)}}\right)^{-\alpha}\right)^{-\beta}\right)\left(1+\left(\frac{G(x ; \xi)}{\overline{G(x ; \xi)}}\right)^{-\alpha}\right)^{-\beta-1}}{\left[1-\left(1-\left(1+\left(\frac{G(x ; \xi)}{\bar{G}(x ; \xi)}\right)^{-\alpha}\right)^{-\beta}\right)^{2}\right]}\right. \\
& \left.\times\left(\frac{G(x ; \xi)}{\bar{G}(x ; \xi)}\right)^{-\alpha} \ln \left(\frac{G(x ; \xi)}{\bar{G}(x ; \xi)}\right)\right) \\
& +\sum_{i=1}^{n} \frac{\beta\left(1+\left(\frac{G(x ; \xi)}{\overline{G(x ; \xi)}}\right)^{-\alpha}\right)^{-\beta-1}\left(\frac{G(x ; \xi)}{\overline{G(x ; \xi)}}\right)^{-\alpha} \ln \left(\frac{G(x ; \xi)}{\overline{G(x ; \xi)}}\right)}{\left(1-\left(1+\left(\frac{G(x ; \xi)}{\bar{G}(x ; \xi)}\right)^{-\alpha}\right)^{-\beta}\right)} \\
& \frac{\partial \ell_{n}}{\partial \beta}=\frac{n}{\beta}+(b-1) \sum_{i=1}^{n} \frac{2\left(1+\left(\frac{G(x ; \xi)}{\bar{G}(x ; \xi)}\right)^{-\alpha}\right)^{-\beta} \ln \left(1+\left(\frac{G(x ; \xi)}{\bar{G}(x ; \xi)}\right)^{-\alpha}\right)}{\left[1-\left(1-\left(1+\left(\frac{G(x ; \xi)}{\bar{G}(x ; \xi)}\right)^{-\alpha}\right)^{-\beta}\right)^{2}\right]} \\
& -\sum_{i=1}^{n} \ln \left(1+\left(\frac{G(x ; \xi)}{\bar{G}(x ; \xi)}\right)^{-\alpha}\right) \text {, } \\
& \frac{\partial \ell_{n}}{\partial b}=\frac{n}{b}+\sum_{i=1}^{n} \ln \left[1-\left(1-\left(1+\left(\frac{G(x ; \xi)}{\bar{G}(x ; \xi)}\right)^{-\alpha}\right)^{-\beta}\right)^{2}\right] \text {, }
\end{aligned}
$$

and

$$
\begin{aligned}
\frac{\partial \ell_{n}}{\partial \xi_{k}} & =\left((b-1) \sum_{i=1}^{n} \frac{2 \alpha \beta\left(1+\left(\frac{G(x ; \xi)}{\overline{G(x ; \xi)}}\right)^{-\alpha}\right)^{-\beta-1}\left(\frac{G(x ; \xi)}{\overline{G(x ; \xi)}}\right)^{-\alpha-1}}{\left[1-\left(1-\left(1+\left(\frac{G(x ; \xi)}{\bar{G}(x ; \xi)}\right)^{-\alpha}\right)^{-\beta}\right)^{2}\right][\bar{G}(x ; \xi)]^{2}}\right. \\
& \left.\times\left(1-\left(1+\left(\frac{G(x ; \xi)}{\bar{G}(x ; \xi)}\right)^{-\alpha}\right)^{-\beta}\right) \frac{\partial g\left(x_{i} ; \xi\right)}{\partial \xi_{k}}\right) \\
& -\sum_{i=1}^{n} \frac{\alpha \beta\left(1+\left(\frac{G(x ; \xi)}{\bar{G}(x ; \xi)}\right)^{-\alpha}\right)^{-\beta-1}\left(\frac{G(x ; \xi)}{\bar{G}(x ; \xi)}\right)^{-\alpha-1} \frac{\partial g\left(x_{i} ; \xi\right)}{\partial \xi_{k}}}{\left(1-\left(1+\left(\frac{G(x ; \xi)}{\bar{G}(x ; \xi)}\right)^{-\alpha}\right)^{-\beta}\right)[\bar{G}(x ; \xi)]^{2}}
\end{aligned}
$$




$$
\begin{aligned}
& +\quad(-\alpha-1) \sum_{i=1}^{n} \frac{\frac{\partial g\left(x_{i} ; \xi\right)}{\partial \xi_{k}}}{\left(\frac{G(x ; \xi)}{\bar{G}(x ; \xi)}\right)[\bar{G}(x ; \xi)]^{2}} \\
& +\quad(\beta+1) \sum_{i=1}^{n} \frac{\alpha\left(\frac{G(x ; \xi)}{\bar{G}(x ; \xi)}\right)^{-\alpha-1} \frac{\partial g\left(x_{i} ; \xi\right)}{\partial \xi_{k}}}{\left(1+\left(\frac{G(x ; \xi)}{\bar{G}(x ; \xi)}\right)^{-\alpha}\right)[\bar{G}(x ; \xi)]^{2}}+\sum_{i=1}^{n} \frac{\frac{\partial g\left(x_{i} ; \xi\right)}{\partial \xi_{k}}}{g(x ; \xi)}-2 \sum_{i=1}^{n} \frac{\frac{\partial \bar{G}(x ; \xi)}{\partial \xi_{k}}}{(\bar{G}(x ; \xi))}
\end{aligned}
$$

The maximum likelihood estimates (MLEs), say $\hat{\boldsymbol{\Delta}}=(\hat{\alpha}, \hat{\beta}, \hat{b}, \hat{\xi})$ can be obtained by equating the system of non linear equations $\frac{\partial \ell_{n}}{\partial \alpha}, \frac{\partial \ell_{n}}{\partial \beta}, \frac{\partial \ell_{n}}{\partial b}, \frac{\partial \ell_{n}}{\partial \xi_{k}}$ to zero and solving simultaneously. These equations cannot be solved analytically as they are not in closed form. Thus, numerical methods such as Newton-Raphson procedure can be used to solve them numerically. We maximize the likelihood function using the function nlm in $\mathrm{R}$ ([30]). The estimated values of the parameters (standard error in parenthesis), -2log-likelihood statistic $(-2 \ln (L)$ ), Akaike Information Criterion $(A I C=2 p-2 \ln (L))$, Bayesian Information Criterion $(B I C=p \ln (n)-2 \ln (L))$ and Consistent Akaike Information Criterion $\left(A I C C=A I C+2 \frac{p(p+1)}{n-p-1}\right)$, where $L=L(\hat{\boldsymbol{\Delta}})$ is the value of the likelihood function evaluated at the parameter estimates, $n$ is the number of observations, and $p$ is the number of estimated parameters are presented. In order to compare the models, we use the criteria stated above. We also obtain the goodness-of-fit statistics: Crameŕ-von Mises $\left(W^{*}\right)$ and Anderson-Darling Statistics $\left(A^{*}\right)$ described by Chen and Balakrishnan [13], as well as Kolmogorov-Smirnov (KS) statistic and its p-value. Note that for AIC, AICC, BIC, and the goodness-of-fit statistics $W^{*}, A^{*}$ and $K S$, smaller values are preferred.

\section{Simulation Study}

The performance of the TL-OBIII-LLoG distribution when $\beta=1$ is examined by conducting various simulations for different sizes $(n=25,50,100,200,400)$ via the $\mathrm{R}$ package. Simulation results for other parameter values are available on request from the authors. We simulate $N=1000$ samples for the true parameters values given in Table 7. The table lists the mean MLEs of the model parameters along with the respective bias and root mean squared errors (RMSEs). The bias and RMSE for the estimated parameter, say, $\hat{\theta}$, say, are given by:

$$
\operatorname{Bias}(\hat{\theta})=\frac{\sum_{i=1}^{N} \hat{\theta}_{i}}{N}-\theta, \quad \text { and } \quad \operatorname{RMSE}(\hat{\theta})=\sqrt{\frac{\sum_{i=1}^{N}\left(\hat{\theta}_{i}-\theta\right)^{2}}{N}}
$$

respectively. 
Table 7. Monte Carlo Simulation Results: Mean, Average Bias and RMSE

\begin{tabular}{|c|c|c|c|c|c|c|c|c|c|c|}
\hline \multirow[b]{2}{*}{ Parameter } & \multirow[b]{2}{*}{$n$} & \multicolumn{3}{|c|}{ I $(1.2,1.0,2.1 .4)$} & \multicolumn{3}{|c|}{ II $(1.0,2.0,2.4)$} & \multicolumn{3}{|c|}{ III $(1.0,3.0,2.0)$} \\
\hline & & Mean & Average Bias & RMSE & Mean & Average Bias & RMSE & Mean & Average Bias & RMSE \\
\hline \multirow[t]{5}{*}{$\alpha$} & 25 & 1.2151 & 0.0151 & 0.1254 & 1.0577 & 0.0577 & 0.1700 & 1.0383 & 0.0383 & 0.1646 \\
\hline & 50 & 1.2105 & 0.0105 & 0.0930 & 1.0347 & 0.0347 & 0.1065 & 1.0377 & 0.0377 & 0.1095 \\
\hline & 100 & 1.2056 & 0.0056 & 0.0649 & 1.0216 & 0.0216 & 0.0745 & 1.01595 & 0.0159 & 0.0690 \\
\hline & 200 & 1.1973 & -0.0027 & 0.0469 & 1.0096 & 0.0096 & 0.0506 & 1.0121 & 0.0121 & 0.0470 \\
\hline & 400 & 1.2017 & 0.0017 & 0.0357 & 1.0037 & 0.0037 & 0.0339 & 1.0085 & 0.0085 & 0.0380 \\
\hline \multirow[t]{5}{*}{$b$} & 25 & 1.0465 & 0.0465 & 0.1831 & 2.0189 & 0.0189 & 0.3054 & 3.1034 & 0.1034 & 0.7515 \\
\hline & 50 & 1.0232 & 0.0232 & 0.1410 & 2.0202 & 0.0202 & 0.2077 & 3.0286 & 0.0286 & 0.4635 \\
\hline & 100 & 1.0163 & 0.0163 & 0.1008 & 2.0030 & 0.0030 & 0.1401 & 2.9788 & -0.0212 & 0.2998 \\
\hline & 200 & 1.0151 & 0.0152 & 0.0668 & 1.9979 & -0.0021 & 0.0978 & 3.0087 & 0.0087 & 0.2200 \\
\hline & 400 & 1.0035 & 0.0035 & 0.0489 & 2.0033 & 0.0033 & 0.0757 & 3.0031 & 0.0031 & 0.1555 \\
\hline \multirow[t]{5}{*}{$\lambda$} & 25 & 1.4122 & 0.0122 & 0.1120 & 2.4087 & 0.0086 & 0.0617 & 2.0148 & 0.0148 & 0.0920 \\
\hline & 50 & 1.4079 & 0.0079 & 0.0818 & 2.4052 & 0.0052 & 0.0421 & 2.0190 & 0.0190 & 0.0739 \\
\hline & 100 & 1.4035 & 0.0035 & 0.0576 & 2.4023 & 0.0024 & 0.0322 & 1.9979 & -0.0020 & 0.0533 \\
\hline & 200 & 1.3969 & -0.0031 & 0.0405 & 2.3994 & -0.0006 & 0.0234 & 1.9853 & -0.0147 & 0.0423 \\
\hline & 400 & 1.4009 & 0.0009 & 0.0306 & 2.3991 & -0.0009 & 0.0159 & 1.9891 & -0.0109 & 0.0359 \\
\hline
\end{tabular}

From the results, we can clearly verify that as the sample size $n$ increases, the mean estimates of the parameters tend to be closer to the true parameter values, since RMSEs decay toward zero.

\section{Applications}

The TL-OBIII-LLoG distribution is fitted to two real data sets and compared to fits of several non-nested four parameter distributions. The TL-OBIII-LLoG distribution is compared to the new modified Weibull (NMW) distribution introduced by Doostmoradi et al. [15], beta generalized exponential (BGE) distribution introduced by Barreto-Souza et al. [6], beta generalized Lindley (BGL) distribution by Oluyede and Yang [29], exponentiated modified Weibull distribution by Elbatal [16], Weibull Lomax (WL) distribution by Tahir et al. [34], generalized Weibull log-logistic (GWLLoG) distribution by Cordeiro et al. [11] and Burr III Marshall Olkin-Lindley (BIIIMOL) distribution by Bhatti et al. [7]. The pdf's of the four parameter NMW, BGE, BGL, EMW, WL, GWLLoG, BIIIMO-L distributions are given in equations (20), (21), (22), (23), (24), (25) and (26) respectively, that is,

$$
\begin{aligned}
g_{N M W}(x) & =\left(\alpha \gamma x^{\gamma-1} e^{\alpha x^{\gamma}}+\lambda \beta x^{\lambda-1} e^{-\beta x^{\lambda}}\right) e^{-e^{\alpha x^{\gamma}}+e^{-\beta x^{\lambda}}}, \quad x>0, \\
g_{B G E}(x)= & \frac{\alpha \lambda}{B(a, b)} e^{-\lambda x}\left(1-e^{-\lambda x}\right)^{\alpha a-1}\left(1-\left(1-e^{-\lambda x}\right)^{\alpha}\right)^{b-1}, \quad x>0, \\
g_{B G L}(x)= & \frac{\alpha \lambda^{2}}{B(a, b)(1+\lambda)}(1+x) e^{-\lambda x}\left[1-\frac{1+\lambda+\lambda x}{1+\lambda} e^{-\lambda x}\right]^{a \alpha-1} \\
& \times\left[1-\left(1-\frac{1+\lambda+\lambda x}{1+\lambda} e^{-\lambda x}\right)^{a}\right]^{b-1}, \quad x>0, \\
g_{E M W}(x)= & \gamma\left[\delta+\lambda \theta^{\alpha} x^{\lambda-1}\right] e^{-\left(\delta x+\left(\theta x^{\lambda}\right)\right.}\left[1-e^{-\left(\delta x+(\theta x)^{\lambda}\right)}\right]^{\delta-1}, \quad x>0,
\end{aligned}
$$




$$
\begin{aligned}
g_{W L}(x) & =\frac{a b \alpha}{\beta}\left[1+\left(\frac{x}{\beta}\right)\right]^{b \alpha-1}\left(1-\left[1+\left(\frac{x}{\beta}\right)\right]^{-\alpha}\right)^{b-1} \\
& \times \exp \left(-a\left(\left[1+\left(\frac{x}{\beta}\right)\right]^{\alpha}-1\right)^{b}\right), \quad x>0, \\
g_{G W L L O G}(x) & =\frac{\alpha \beta \theta x^{\theta-1}}{a^{\theta}} \exp \left(-\alpha\left[\log \left(1+\left(\frac{x}{a}\right)^{\theta}\right)\right]^{\beta}\right) \\
& \times\left(1+\left(\frac{x}{a}\right)^{\theta}\right)^{-1}\left[\log \left(1+\left(\frac{x}{a}\right)^{\theta}\right)\right]^{\beta-1}, \quad x>0,
\end{aligned}
$$

and

$$
\begin{aligned}
g_{\text {BIIIMO-L }}(x) & =\left(-\log \left[\frac{\lambda\left(1+\frac{b x}{1+b}\right) \exp (-b x)}{1-\bar{\lambda}\left(1+\frac{b x}{1+b}\right) \exp (-b x)}\right]\right)^{-\beta-1} \\
& \times\left(1+\left(-\log \left[\frac{\lambda\left(1+\frac{b x}{1+b}\right) \exp (-b x)}{1-\bar{\lambda}\left(1+\frac{b x}{1+b}\right) \exp (-b x)}\right]\right)^{-\beta}\right)^{-\alpha-1}, \quad x>0 . \\
& \times \frac{\frac{\alpha \beta b^{2}(1+x) \exp (-b x)}{1+b}}{\left(1+\frac{b x}{1+b}\right) \exp (-b x)}, \quad x>0 .
\end{aligned}
$$

Plots of the fitted densities, the histogram of the data and probability plots (Chambers et al. [12]) are given in Figure 4 and Figure 5 for the two data sets considered in this section. For the probability plot, we plotted $F_{T L-O B I I-L L o G}\left(x_{(j)} ; \hat{\alpha}, \hat{\beta}, \hat{b}, \hat{\lambda}\right)$ against $\frac{j-0.375}{n+0.25}, j=1,2, \cdots, n$, where $x_{(j)}$ are the ordered values of the observed data. The measures of closeness are given by the sum of squares (SS) as $S S=\sum_{j=1}^{n}\left[F_{T L-O B I I I-L L O G}\left(x_{(j)} ; \hat{\alpha}, \hat{\beta}, \hat{b}, \hat{\lambda}\right)-\left(\frac{j-0.375}{n+0.25}\right)\right]^{2}$.

The goodness-of-fit statistics $W^{*}$ and $A^{*}$, described by Chen and Balakrishnan [13] as well as KolmogorovSmirnov (KS) statistic, its P-value and SS are also presented in the tables. These statistics can be used to verify which distribution fits better to the data. In general, the smaller the values of $W^{*}$ and $A^{*}$ and $\mathrm{K}-\mathrm{S}$, the better the fit.

\subsection{Growth Hormone Data}

The first data consists of the estimated time since growth hormone medication until the children reached the target age. The data was used by Alizadeh et al. [4] to show the superiority of the exponentiated power Lindley power series (EPLPS) class of distributions distributions. The data are:

$2.15,2.20,2.55,2.56,2.63,2.74,2.81,2.90,3.05,3.41,3.43,3.43,3.84,4.16,4.18,4.36,4.42,4.51,4.60,4.61$, $4.75,5.03,5.10,5.44,5.90,5.96,6.77,7.82,8.00,8.16,8.21,8.72,10.40,13.20,13.70$.

Estimates of the parameters of TL-OBIII-LLoG distribution and the non-nested models (standard error in parentheses), AIC, BIC, and the goodness-of-fit statistics $\mathrm{W}^{*}, \mathrm{~A}^{*}, \mathrm{KS}$ and its p-value as well as SS are given in Table 8. Plots of the fitted densities and the histogram, observed probability vs predicted probability are given in Figure 4. 
Table 8. Estimates of Models for Growth Hormone Data

\begin{tabular}{|c|c|c|c|c|c|c|c|c|c|c|c|c|c|}
\hline \multirow[b]{2}{*}{ Model } & \multicolumn{4}{|c|}{ Estimates } & \multicolumn{9}{|c|}{ Statistics } \\
\hline & $\alpha$ & $\beta$ & $\mathrm{b}$ & $\lambda$ & $-2 \log L$ & $A I C$ & $A I C C$ & $B I C$ & $W^{*}$ & $A^{*}$ & $K S$ & P-value & $S S$ \\
\hline TL-OBIII-LLoG & $\begin{array}{c}0.0710 \\
\left(8.4675 \times 10^{-03}\right)\end{array}$ & $\begin{array}{c}0.5180 \\
\left(9.9284 \times 10^{-02}\right)\end{array}$ & $\begin{array}{c}168.7000 \\
\left(1.6615 \times 10^{-04}\right)\end{array}$ & $\begin{array}{c}19.0390 \\
\left(3.3992 \times 10^{-05}\right)\end{array}$ & 155.4418 & 163.4418 & 164.7751 & 169.6632 & 0.0389 & 0.2685 & 0.0873 & 0.9522 & 0.0389 \\
\hline & $\alpha$ & $\lambda$ & $a$ & $b$ & & & & & & & & & \\
\hline BGE & $\begin{array}{c}0.7487 \\
(0.5541)\end{array}$ & $\begin{array}{c}0.0323 \\
(0.0111)\end{array}$ & $\begin{array}{c}6.3824 \\
(7.1232)\end{array}$ & $\begin{array}{l}19.9198 \\
(0.5568)\end{array}$ & 159.7534 & 167.7534 & 169.0867 & 173.9748 & 0.0944 & 0.6166 & 0.1184 & 0.7097 & 0.0907 \\
\hline & $\alpha$ & $\gamma$ & $\lambda$ & $\beta$ & & & & & & & & & \\
\hline NMW & $\begin{array}{c}0.0006 \\
(0.0014)\end{array}$ & $\begin{array}{l}2.8548 \\
(0.8086)\end{array}$ & $\begin{array}{l}4.0927 \\
(0.7964)\end{array}$ & $\begin{array}{c}0.0018 \\
(0.0021)\end{array}$ & 168.0796 & 176.0796 & 177.413 & 182.301 & 0.0551 & 0.3873 & 0.1618 & 0.3187 & 0.1439 \\
\hline & $\alpha$ & $\theta$ & $a$ & $b$ & & & & & & & & & \\
\hline BGL & $\begin{array}{c}0.1179 \\
\left(9.1449 \times 10^{-03}\right) \\
\gamma\end{array}$ & $\begin{array}{c}1.1290 \times 10^{-07} \\
\left(2.4083 \times 10^{-06}\right) \\
\delta\end{array}$ & $\begin{array}{c}3.0140 \times 10^{-01} \\
\left(8.1743 \times 10^{-04}\right) \\
\lambda\end{array}$ & $\begin{array}{c}1.0100 \times 10^{01} \\
\left(2.6267 \times 10^{-05}\right) \\
\theta\end{array}$ & 342.7928 & 350.8082 & 352.1415 & 357.0296 & 0.0685 & 0.4632 & 0.4833 & $1.579 \times 10^{-07}$ & 2.6551 \\
\hline EMW & $\begin{array}{c}6.5130 \\
(2.2693) \\
a\end{array}$ & $\begin{array}{c}0.4820 \\
(0.07981) \\
b\end{array}$ & $\begin{array}{c}17.0010 \\
\left(1.7949 \times 10^{-16}\right) \\
\alpha\end{array}$ & $\begin{array}{c}1.0000 \times 10^{-04} \\
\left(3.0495 \times 10^{-11}\right) \\
\beta\end{array}$ & 158.1951 & 166.1951 & 167.5284 & 172.4165 & 0.0703 & 0.4747 & 0.1012 & 0.8656 & 0.0754 \\
\hline WL & $\begin{array}{c}0.0002 \\
(0.0003) \\
\alpha\end{array}$ & $\begin{array}{c}4.3897 \\
(0.0111) \\
\beta\end{array}$ & $\begin{array}{c}0.3987 \\
(0.0653) \\
a\end{array}$ & $\begin{array}{c}0.0380 \\
(0.0507) \\
\theta\end{array}$ & 164.004 & 172.0040 & 173.3373 & 178.2254 & 0.1508 & 0.9496 & 0.1504 & 0.4069 & 0.1533 \\
\hline GWLLoG & $\begin{array}{c}0.2962 \\
(0.5048) \\
\alpha\end{array}$ & $\begin{array}{c}2.7285 \\
(1.3142) \\
\beta\end{array}$ & $\begin{array}{c}2.3479 \\
(1.6100) \\
\lambda\end{array}$ & $\begin{array}{c}1.4831 \\
(0.7834) \\
b\end{array}$ & 159.4803 & 167.4803 & 168.8137 & 173.7017 & 0.0863 & 0.5686 & 0.1158 & 0.7352 & 0.0829 \\
\hline BIIIMO-L & $\begin{array}{l}0.1177 \\
(0.0205)\end{array}$ & $\begin{array}{c}0.1109 \\
(0.0168)\end{array}$ & $\begin{array}{c}0.1097 \\
(0.0185)\end{array}$ & $\begin{array}{c}5.8707 \times 10^{-06} \\
\left(3.3596 \times 10^{-05}\right)\end{array}$ & 556.1171 & 564.1172 & 565.4505 & 570.3386 & 0.0723 & 0.4860 & 0.8985 & $2.2000 \times 10^{-16}$ & 8.8178 \\
\hline
\end{tabular}

The values in the table above showed that the TL-OBIII-LLoG distribution has the smallest values of AIC, AICC, BIC, $\mathrm{W}^{*}, \mathrm{~A}^{*}, \mathrm{KS}$ and the largest $\mathrm{p}$-value compared to the corresponding values for the fitted non-nested models. So, we conclude that the TL-OBIII-LLoG distribution could be chosen as the best fitting model for the growth hormone data.
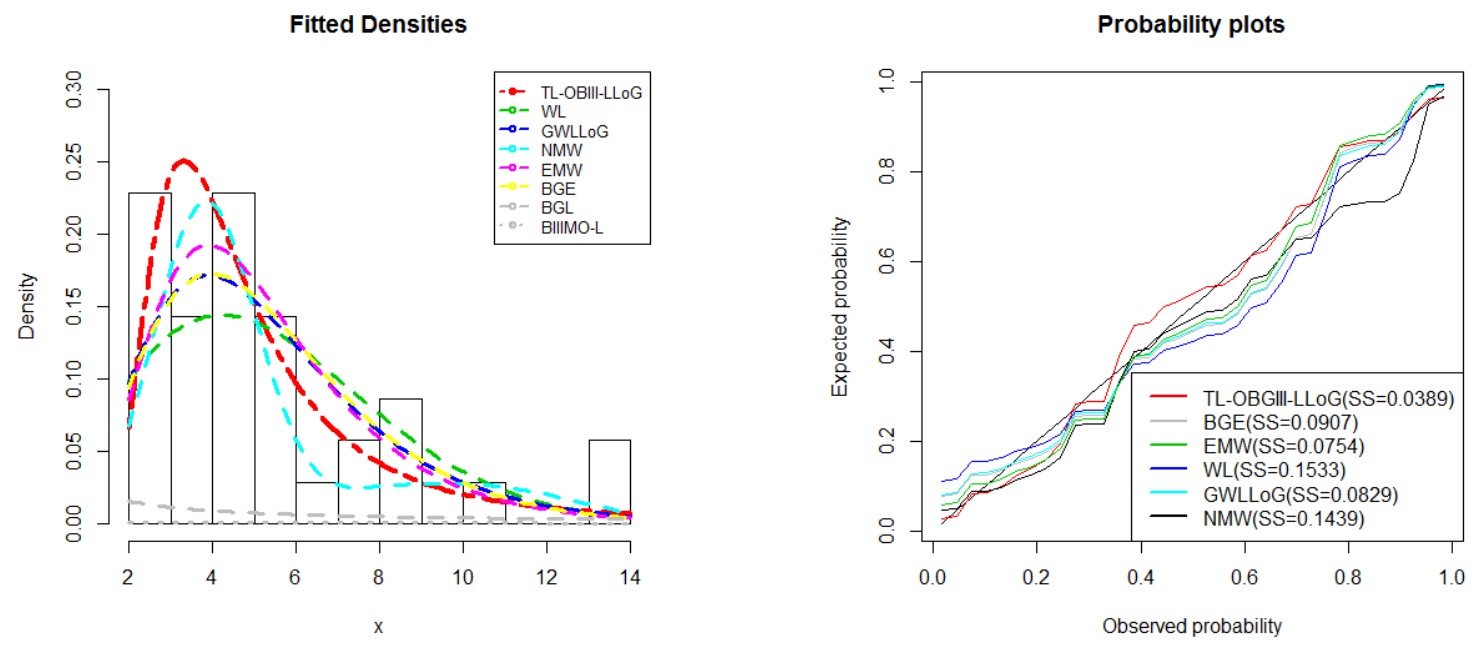

Figure 4. Fitted Densities and Probability Plots of the Growth Hormone Data

\subsection{Repair Lifetimes of an Airborne Transceiver Data}

The second data correspond to maintenance on active repair times (in hours) for an airborne communication transceiver with size $n=46$ from Leiva et al. [23] and Chhikara and Folks [14]. These data are:

$0.2,0.3,0.5,0.5,0.5,0.5,0.6,0.6,0.7,0.7,0.7,0.8,0.8,1.0,1.0,1.0,1.0,1.1,1.3,1.5,1.5,1.5,1.5,2.0,2.0,2.2$, 2.5, 2.7, 3.0, 3.0, 3.3, 3.3, 4.0, 4.0, 4.5, 4.7, 5.0, 5.4, 5.4, 7.0, 7.5, 8.8, 9.0, 10.3, 22.0, 24.5.

Estimates of the parameters of TL-OBIII-LLoG distribution and non-nested models (standard error in parentheses), 
AIC, BIC, and the goodness-of-fit statistics $\mathrm{W}^{*}, \mathrm{~A}^{*}, \mathrm{KS}$ and its p-value as well as SS are given in Table 9. Plots of the fitted densities and the histogram, observed probability vs predicted probability are given in Figure 5.

Table 9. Estimates of Models for Repair Lifetimes of an Airborne Transceiver Data

\begin{tabular}{|c|c|c|c|c|c|c|c|c|c|c|c|c|c|}
\hline \multirow{2}{*}{ Model } & \multicolumn{4}{|c|}{ Estimates } & \multicolumn{9}{|c|}{ Statistics } \\
\hline & $\alpha$ & $\beta$ & $\mathrm{b}$ & $\lambda$ & $-2 \log L$ & $A I C$ & $A I C C$ & $B I C$ & $W^{*}$ & $A^{*}$ & $K S$ & P-value & $S S$ \\
\hline \multirow[t]{3}{*}{ TL-OBIII-LLoG } & 0.4601 & 0.1392 & 144.0183 & 1.5403 & 199.9626 & 207.9626 & 208.9382 & 215.2772 & 0.0515 & 0.3127 & 0.0762 & 0.9520 & 0.0508 \\
\hline & ( 15.4843$)$ & $(0.0108)$ & $(0.0043)$ & (51.8267) & & & & & & & & & \\
\hline & $\alpha$ & $\lambda$ & $a$ & $b$ & & & & & & & & & \\
\hline \multirow[t]{3}{*}{ BGE } & $\begin{array}{c}10.9759 \\
(20.9289)\end{array}$ & $\begin{array}{c}1.2799 \\
(07340)\end{array}$ & $\begin{array}{l}0.1848 \\
(03193)\end{array}$ & $\begin{array}{l}0.1855 \\
(0.0620)\end{array}$ & 201.8082 & 209.8083 & 210.7839 & 217.1229 & 0.0563 & 0.4558 & 0.1109 & 0.6228 & 0.0890 \\
\hline & (20.9289) & $(0.7340)$ & $(0.3193)$ & $(0.0620)$ & & & & & & & & & \\
\hline & $\alpha$ & $\gamma$ & $\lambda$ & $\beta$ & & & & & & & & & \\
\hline \multirow[t]{2}{*}{ NMW } & $\begin{array}{c}0.0377 \\
(0.0333)\end{array}$ & $\begin{array}{c}1.0873 \\
(0.2863)\end{array}$ & $\begin{array}{l}1.3624 \\
(0.2228)\end{array}$ & $\begin{array}{c}0.2518 \\
(0.0803)\end{array}$ & 218.5555 & 226.5555 & 227.5311 & 233.8701 & 0.0681 & 0.4514 & 0.1604 & 0.1870 & 0.3884 \\
\hline & $\alpha$ & $\theta$ & $a$ & $b$ & & & & & & & & & \\
\hline \multirow[t]{2}{*}{ BGL } & $\begin{array}{c}0.1179 \\
\left(8.1272 \times 10^{-03}\right)\end{array}$ & $\begin{array}{c}1.3719 \times 10^{-07} \\
\left(2.4697 \times 10^{-06}\right)\end{array}$ & $\begin{array}{c}0.3014 \\
\left(7.7978 \times 10^{-04}\right)\end{array}$ & $\begin{array}{c}10.1000 \\
\left(22593 \times 10^{-05}\right)\end{array}$ & 382.0483 & 390.1551 & 391.1307 & 397.4696 & 0.0837 & 0.5519 & 0.4630 & $5.4390 \times 10^{-09}$ & 3.2424 \\
\hline & $\gamma$ & $\delta$ & $\lambda$ & $\theta$ & & & & & & & & & \\
\hline \multirow[t]{3}{*}{ EMW } & 0.9582 & 0.2693 & $\begin{array}{c}17.0010 \\
\left(34209 \times 10^{-18}\right.\end{array}$ & $1.0000 \times 10^{-04}$ & 209.9658 & 217.9658 & 218.9414 & 225.2804 & 0.1441 & 1.0004 & 0.1519 & 0.2385 & 0.1889 \\
\hline & $(0.1897)$ & $(0.0543)$ & $\left(3.4209 \times 10^{-18}\right)$ & $\left(5.8159 \times 10^{-13}\right)$ & & & & & & & & & \\
\hline & $a$ & $b$ & $\alpha$ & $\beta$ & & & & & & & & & \\
\hline \multirow[t]{2}{*}{ WL } & $\begin{array}{c}1.6956 \mathrm{e}+03 \\
(7.6319 \mathrm{e}-05)\end{array}$ & $\begin{array}{c}2.5654 \\
(1.3635)\end{array}$ & $\begin{array}{c}0.0198 \\
(0.0158)\end{array}$ & $\begin{array}{c}0.2078 \\
(0.4281)\end{array}$ & 200.603 & 208.603 & 209.5786 & 215.9176 & 0.0563 & 0.3634 & 0.0937 & 0.8132 & 0.0509 \\
\hline & $\alpha$ & $\beta$ & $a$ & $\theta$ & & & & & & & & & \\
\hline \multirow[t]{3}{*}{ GWLLoG } & 8.2995 & 0.1697 & 35.4344 & 5.3096 & 208.9809 & 216.9809 & 217.9565 & 224.2954 & 0.1295 & 0.8973 & 0.1199 & 0.5218 & 0.1147 \\
\hline & ( 1.8675$)$ & $(0.1866)$ & (1.2987) & (5.7670) & & & & & & & & & \\
\hline & $\alpha$ & $\beta$ & $\lambda$ & $b$ & & & & & & & & & \\
\hline BIIIMO-L & $\begin{array}{c}0.1184 \\
(0.0187)\end{array}$ & $\begin{array}{l}0.1121 \\
(0.0175)\end{array}$ & $\begin{array}{c}0.1107 \\
(0.0163)\end{array}$ & $\begin{array}{c}1.1385 \times 10^{-05} \\
\left(1.8930 \times 10^{-05}\right)\end{array}$ & 659.9823 & 667.9820 & 668.9577 & 675.2966 & 0.0902 & 0.6006 & 0.8711 & $2.2000 \times 10^{-16}$ & 11.4417 \\
\hline
\end{tabular}

In Table 9 the values of AIC, AICC, BIC, $\mathrm{W}^{*}, \mathrm{~A}^{*}$, KS for TL-OBIII-LLoG distribution are smaller than those for fitted non-nested distributions, which indicates that the TL-OBIII-LLoG distribution provides a better fit for the repair lifetimes of an airborne transceiver data. Also, we can conclude that the TL-OBIII-LLoG distribution is the better fit from the p-value of the KS statistic as it is large for TL-OBIII-LLoG distribution as compared to those from the other non-nested distributions for the repair lifetimes of an airborne transceiver data.
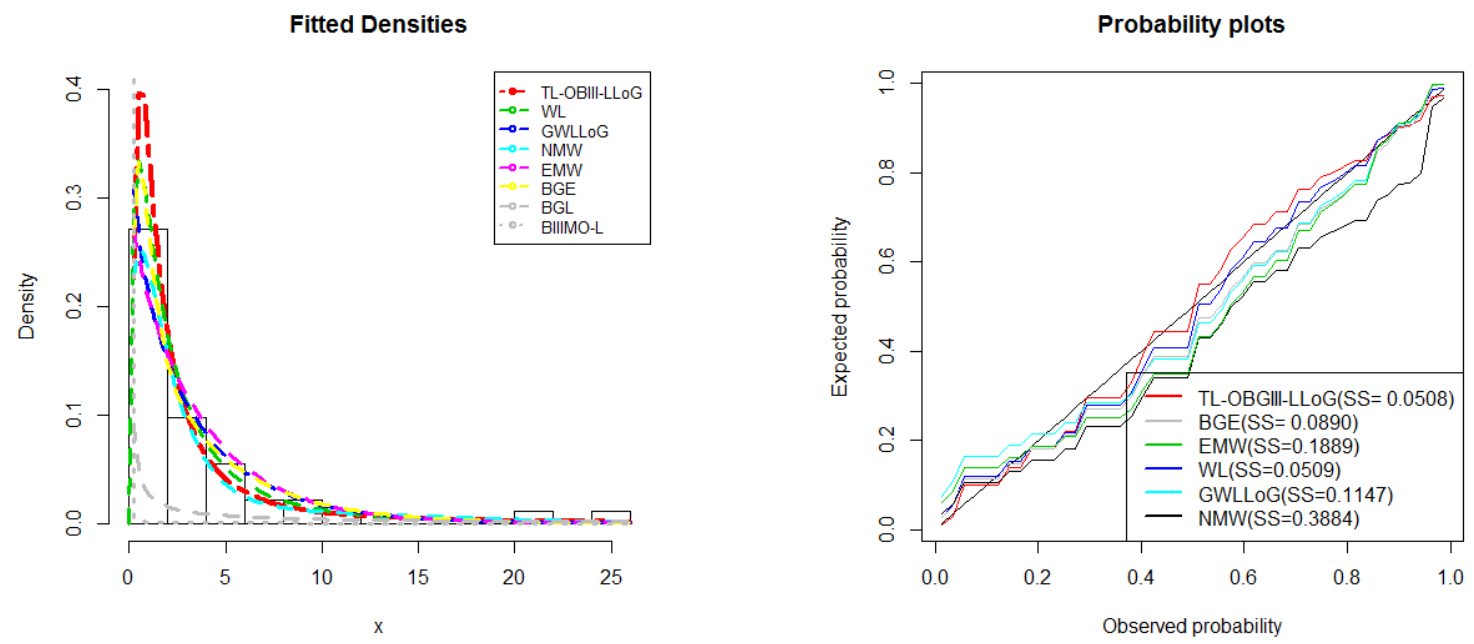

Figure 5. Fitted Densities and Probability Plots of the Repair Lifetimes of an Airborne Transceiver Data 


\section{Concluding Remarks}

We have developed a new family of distributions called the Topp-Leone Odd Burr III-G (TL-OBIII-G) family of distributions. Properties of this new distribution such as sub-models, quantile function, moments, incomplete moments, probability weighted moments, order statistics and Rényi entropy were studied. The maximum likelihood estimates (MLEs) have been computed via the maximum likelihood estimation method. Monte Carlo simulation study was employed to check the perfomance of the MLEs. Lastly, we illustrated that the TL-OBIII-G family of distributions is useful for lifetime applications by fitting its special case of TL-OBIII-LLoG distribution to two real data sets.

\section{REFERENCES}

1. Abbas, S., Taqi, S. A., Mustafa, F., Murtaza, M., and Shahbaz, M. Q., Topp-Leone Inverse Weibull Distribution: Theory and Application, European Journal of Pure And Applied Mathematics, vol. 10, no. 5, pp. 1005-1022, 2017.

2. Afify, A. Z., Cordeiro G., Jamal, F., Elgarhy, M., and Nasir, M., The Marshall-Olkin Odd Burr III-G Family of Distributions: Theory, Estimation and Applications, hal-02376067, 2019.

3. Al-Shomrani, A., Arif, O., Shawky, A., Hanif, S., and Shahbaz, M. Q., Topp-Leone Family of Distributions: Some Properties and Application, Pakistan Journal of Statistics and Operation Research, vol. 12, no. 3, pp. 443-451, 2016.

4. Alizadeh, M., Bagheri, S. F., Bahrami S. E., Ghobadi, S., and Nadarajah, S., Exponentiated Power Lindley Power Series Class of Distributions: Theory and Applications, Communications in Statistics-Simulation and Computation, 2017.

5. Aryal, G. R., Ortega, E. M., Hamedani, G. G., and Yousof, H. M., The Topp-Leone Generated Weibull Distribution: Regression Model, Characterizations and Applications, International Journal of Statistics and Probability, vol. 6, no. 1, 2017.

6. Barreto-Souza, W., Santos, A. H. S. and Cordeiro, G. M., The Beta Generalized Exponential Distribution, Journal of Statistical Computation and Simulation, vol. 80, no. 2, pp. 159-172, 2010.

7. Bhatti F. A., Hamedani, G. G., Korkmaz, M. C., Cordeiro, G. M., Yousof, H. M., and Ahmad, M., On Burr III Marshal-Olkin Family: Development, Properties, Characterizations and Applications, Journal of Statistical Distributions and Applications, vol. 6, no. 12, 2019.

8. Burr, I. W., Cumulative Frequency Functions, Annals of Mathematical Statistics, vol. 13, pp. 215-232, 1942.

9. Cordeiro, G. M., Afify, A. Z., Ortega, E. M. M., Suzuki, A. K. and Mead, M. E., The Odd Lomax Generator of Distributions: Properties, Estimation and Applications, Journal of Computational and Applied Mathematics, vol. 347, pp. 222-237, 2019.

10. Cordeiro, G. M., Afify, A. Z., Yousof, H. M., Pescim, R. R., and Arya, G. R., The Exponentiated Weibull-H Family of Distributions: Theory and Applications, Mediterranean Journal of Mathematics, vol. 14, no. 155, 2017.

11. Cordeiro, G. M., Ortega, E. M. M., and Ramires T. G., A New Generalized Weibull Family of Distributions: Mathematical Properties and Applications, Journal of Statistical Distributions and Applications, vol. 2, no. 13, 2015.

12. Chambers, J., Cleveland, W., Kleiner, B., and Tukey, J., Graphical Methods for Data Analysis, Chapman and Hall, London, 1983.

13. Chen, G. and Balakrishnan, N., A General Purpose Approximate Goodness-of-Fit Test, Journal of Quality Technology, vol. 27, pp. 154-161, 1995

14. Chhikara R. S., and Folks J. I., (1977). The Inverse Gaussian Distribution as a Lifetime Model, Technometrics, vol. 19, no. 4 pp.461-468.

15. Doostmoradi, A., Zadkarami, M. R., and Roshani Sheykhabad, A., A New Modified Weibull Distribution and Its Application, Journal of Statistical Research and Training Center, Iran vol. 11, pp. 97-118, 2014.

16. Elbatal, I., Exponentiated Modified Weibull Distribution. Economic Quality Control, vol. 26, pp. 189-200, 2011.

17. Eugene, N., Lee, C., and Famoye, F., Beta-Normal Distribution and its Applications, Communications in Statistics: Theory and Methods, vol. 31, pp. 497-512, 2002.

18. Gove, J. H., Ducey, M. J., Leak, W. B., and Zhang, L., Rotated Sigmoid Structures in Managed Uneven-aged Northern Hardwood Stands: A Look at the Burr Type III Distribution, Forestry, 2008.

19. Jamal, F., Nasir, M. A., Tahir, M. H., and Montazeri, N. H., The Odd Burr-III Family of Distributions, Journal of Statistics Applications and Probability, vol. 6, no. 1, pp. 105-122, 2017.

20. Jamal, F., Reyad, H. M., Nasir, M. A., Chesneau, C., Shah, M. A. A., and Ahmed, S. O., Topp-Leone Weibull-Lomax distribution: Properties, Regression Model and Applications, hal-02270561f, 2019.

21. Klugman, S. A., Panjer, H. H., and Willmot, G. E., Loss Models, Wiley, NewYork, 1998.

22. Kunjiratanachot, N., Bodhisuwan, W., and Volodin, A., The Topp-Leone Generalized Exponential Power Series Distribution with Applications, Journal of Probability and Statistical Science, vol. 16, no. 2, pp. 197-208, 2018.

23. Leiva V., Barros M., and Paula G. A., Generalized Birnbaum-Sanders Models using R, Brazilian Statistical Association, vol. 53, pp. 1511-1528, 2009.

24. Lindsay, S. R. ,Wood, G. R., and Woollons, R. C., Modelling the Diameter Distribution of Forest Stands Using the Burr Distribution, Journal of Applied Statistics, vol. 23, pp. 609-619, 1996.

25. Mielke, P. W., Another Family of Distributions for Describing and Analyzing Precipitation Data, Journal of Applied Meterology, vol. 12, pp. 275-280, 1973

26. Mohammed, H. F., and Yahia N., On Type II Topp-Leone Inverse Rayleigh Distribution, Applied Mathematical Sciences, vol. 13, no. 13, pp. 607-615, 2019. 
27. Nassar, M., Kumar, D., Dey, S., Cordeiro, G. M., and Afify, A. Z., The Marshall-Olkin Alpha Power Family of Distributions with Applications, Journal of Computational and Applied Mathematics, vol. 351, pp. 41-53, 2019.

28. Nofal, Z. M., Afify, A. Z., Yousof, H. M., and Cordeiro G. M., The Generalized Transmuted-G Family of Distributions, Communications in Statistics Theory and Methods, vol. 46, pp. 4119-4136, 2016

29. Oluyede, B. O, and Yang, T., A New Class of Generalized Lindley Distributions with Applications, Journal of Statistical Computation and Simulation, vol. 85, no. 10, pp. 2072-2100, 2015.

30. R Development Core Team, A Language and Environment for Statistical Computing, R Foundation for Statistical Computing, Vienna, Austria, 2011.

31. Rényi, A., On Measures of Entropy and Information, Proceedings of the Fourth Berkeley Symposium on Mathematical Statistics and Probability, vol. 1, pp. 547-561, 1960.

32. Reyad, H., Alizadeh, M., Jamal, F. and Othman, S., The Topp-Leone Odd Lindley-G Family of Distributions: Properties and Applications, Journal of Statistics and Management Systems, vol. 21, no. 7, pp. 1273-1297, 2018.

33. Reyad, H., Selim, M. A., and Othman, S., The Nadarajah Haghighi Topp-Leone-G Family of Distributions with Mathematical Properties and Application, Pakistan Journal of Statistics and Operation Research, vol. XV, no. IV, pp. 849-866, 2019.

34. Tahir, M. H., Cordeiro, G. M., Mansoor, M. and Zubair, M., The Weibull Lomax Distribution: Properties and Applications, Hacettepe Journal of Mathematics and Statistics, vol. 44, no. 2, pp. 461-480, 2015. 